\title{
Dielectron production in proton-proton and proton-lead collisions at $\sqrt{s_{\mathrm{NN}}}=5.02 \mathrm{TeV}$
}

\author{
S. Acharya et al.* \\ (ALICE Collaboration)
}

(Received 6 August 2020; accepted 21 October 2020; published 25 November 2020)

\begin{abstract}
The first measurements of dielectron production at midrapidity $\left(\left|\eta_{\mathrm{e}}\right|<0.8\right)$ in proton-proton and proton-lead collisions at $\sqrt{s_{\mathrm{NN}}}=5.02 \mathrm{TeV}$ at the LHC are presented. The dielectron cross section is measured with the ALICE detector as a function of the invariant mass $m_{\mathrm{ee}}$ and the pair transverse momentum $p_{\mathrm{T}, \mathrm{ee}}$ in the ranges $m_{\mathrm{ee}}<3.5 \mathrm{GeV} / c^{2}$ and $p_{\mathrm{T}, \mathrm{ee}}<8 \mathrm{GeV} / c$, in both collision systems. In proton-proton collisions, the charm and beauty cross sections are determined at midrapidity from a fit to the data with two different event generators. This complements the existing dielectron measurements performed at $\sqrt{s}=7$ and $13 \mathrm{TeV}$. The slope of the $\sqrt{s}$ dependence of the three measurements is described by FONLL calculations. The dielectron cross section measured in proton-lead collisions is in agreement, within the current precision, with the expected dielectron production without any nuclear matter effects for $\mathrm{e}^{+} \mathrm{e}^{-}$pairs from open heavy-flavor hadron decays. For the first time at LHC energies, the dielectron production in proton-lead and proton-proton collisions are directly compared at the same $\sqrt{s_{\mathrm{NN}}}$ via the dielectron nuclear modification factor $R_{\mathrm{pPb}}$. The measurements are compared to model calculations including cold nuclear matter effects, or additional sources of dielectrons from thermal radiation.
\end{abstract}

DOI: 10.1103/PhysRevC.102.055204

\section{INTRODUCTION}

ALICE [1], located at the Large Hadron Collider (LHC) at CERN, was designed to study the quark-gluon plasma (QGP), a state of matter which consists of deconfined quarks and gluons. The QGP is created at the high-energy densities and temperatures reached in ultra-relativistic heavy-ion collisions. Under these conditions, the chiral symmetry is expected to be restored in the QGP phase [2,3]. Dileptons $\left(1^{+} 1^{-}\right.$, i. e., $\mathrm{e}^{+} \mathrm{e}^{-}$or $\mu^{+} \mu^{-}$) are emitted during all stages of the heavy-ion collision and carry information about the medium properties at the time of their emission, as they do not interact strongly. This makes them a very promising tool to understand the chiral symmetry restoration and the thermodynamical properties of the QGP. In particular, the measurement of the dilepton invariant mass $\left(m_{11}\right)$ allows for the separation of the different stages of the medium evolution. For $m_{\mathrm{ee}}<1.1 \mathrm{GeV} / c^{2}$, the main dilepton sources are Dalitz decays of pseudoscalar mesons $\left(\pi^{0}, \eta, \eta^{\prime}\right)$ as well as Dalitz and two-body decays of vector mesons $(\rho$, $\omega, \phi)$. In this mass range, the dilepton spectrum is sensitive to the in-medium modification of the $\rho$ meson spectral function, which is connected to the partial restoration of chiral symmetry in the hot hadronic phase [3,4]. At the same time, thermal radiation from the medium, contributing over a broad mass range, provides insight into the temperature of the medium and its space-time evolution.

*Full author list given at the end of the article.

Published by the American Physical Society under the terms of the Creative Commons Attribution 4.0 International license. Further distribution of this work must maintain attribution to the author(s) and the published article's title, journal citation, and DOI.
Measurements of dilepton production in nucleus-nucleus collisions were performed at the Super Proton Synchroton at CERN, among others, by CERES [5,6] and NA60 [7] at a center-of-mass energy per nucleon-nucleon pair, $\sqrt{s_{\mathrm{NN}}} \leqslant 17.3 \mathrm{GeV}$. An excess of dileptons compared to the expectation from known hadron decays was observed. It can be ascribed to thermal sources, primarily from thermal production of $\rho$ mesons for $m_{\mathrm{ll}}<1 \mathrm{GeV} / c^{2}$ with a strongly broadened $\rho$ spectral function [7], as well as partonic thermal radiation for $m_{\mathrm{ll}}>1 \mathrm{GeV} / c^{2}$ [8]. At higher energies $\sqrt{s_{\mathrm{NN}}}=200 \mathrm{GeV}$, results from PHENIX [9] and STAR [10] at the Relativistic Heavy Ion Collider (RHIC) are also compatible with models involving a broadening of the $\rho$ spectral function. The study of thermal radiation from the QGP in the intermediate-mass region (IMR), $1.1<m_{11}<2.7 \mathrm{GeV} / c^{2}$, is however challenging at these center-of-mass energies due to the large background from correlated $\mathrm{I}^{+} \mathrm{l}^{-}$pairs originating from open heavy-flavor hadron decays. The first measurement of low- $m_{11}$ dileptons at the LHC, performed by ALICE in $\mathrm{Pb}-\mathrm{Pb}$ collisions at $\sqrt{s_{\mathrm{NN}}}=2.76 \mathrm{TeV}$ [11], does not provide data sensitive to a thermal signal due to the limited statistical precision and the limited knowledge of the charm contribution. Consequently, it is crucial to understand the dilepton production in proton-proton (pp) collisions, in particular the contribution from heavy-flavor hadron decays, to single out the characteristic signals of the QGP.

In pp collisions, the production of charm and beauty quarks can be estimated with perturbative quantum chromodynamics (pQCD) calculations in vacuum without any initial- and finalstate effects. Owing to flavor conservation, the heavy quarks can only be produced in pairs. The resulting lepton pairs originating from charm hadron decays reflect the initial kinematic correlations between the charm and the anti-charm quarks, whereas in the case of beauty hadron decays the correlation 
is weakened because of their large masses. In pp collisions, where no thermal dilepton sources are expected, the $1^{+} 1^{-}$pairs arising from heavy-flavor hadron decays are the main contribution to the dilepton yield in the IMR. Hence, dileptons can be used to study the heavy-quark production mechanisms. Together with the measurements of single heavy-flavor hadrons and their decay products, accurate results on dilepton production can provide constraints on the Monte Carlo (MC) event generators aiming to describe heavy-flavor production. In studies with dielectrons by PHENIX in pp collisions at $\sqrt{s_{\mathrm{NN}}}=200 \mathrm{GeV}[12,13]$, and more recently by ALICE in pp collisions at $\sqrt{s}=7$ and $13 \mathrm{TeV}[14,15]$ the charm and beauty cross sections at midrapidity and in the full phase space were extracted by means of the analysis of the dielectron invariant mass $\left(m_{\mathrm{ee}}\right)$ and pair transverse momentum $\left(p_{\mathrm{T}, \mathrm{ee}}\right)$ spectra. The measured cross sections at the LHC and RHIC were found to be consistent with fixed order plus next-toleading logarithms (FONLL) calculations [16].

The production of dileptons in heavy-ion collisions can be modified with respect to pp collisions not only by the presence of hot nuclear matter but also by the presence of cold nuclear matter (CNM). The CNM effects include the modification of the quark and gluon content in the initial state, that is described by means of parton distribution functions (PDFs) of the incoming nucleons in the collinear factorization framework. In nucleons that are bound in the nucleus, the PDFs are altered by the presence of additional nuclear matter with respect to free nucleons. This modification depends on the parton momentum fraction $x$, the atomic mass number of the nucleus $A$, and the momentum transfer $Q^{2}$ in the hard scattering process. Nuclear PDFs are obtained from a global fit to data from different experiments [17-19]. When the phase space density of gluons within the hadron is high due to gluon self-interactions, reaching a saturation regime, an appropriate theoretical description is the color glass condensate (CGC) theory [20-23]. At LHC energies at midrapidity, where small values of $x$ are probed by the charm and beauty production $\left(x \leqslant 10^{-3}\right)$, the most relevant effect on the PDFs is shadowing [24]. The modification of the initial state in hadronic collisions can significantly reduce the heavy-flavor production cross sections at low transverse momentum $\left(p_{\mathrm{T}}\right)$. In addition, multiple scattering of partons in the nucleus, before and/or after the hard scattering, can change the kinematic distribution of the produced hadrons and affect their azimuthal correlation, such that the $m_{\mathrm{ee}}$ and $p_{\mathrm{T}, \mathrm{ee}}$ distributions from correlated heavy-flavor hadron decays could be modified [25,26].

Initially, hot matter effects were not expected in proton-nucleus (pA) collisions, so they were used as a baseline for measurements in heavy-ion collisions to study possible CNM effects. At LHC energies in minimum bias (MB) $\mathrm{p}-\mathrm{Pb}$ collisions at midrapidity, the measured $p_{\mathrm{T}}$ differential production cross sections of single open-charm hadrons $[27,28]$ and their decay electrons $[29,30]$, as well as results on azimuthal correlations of $\mathrm{D}$ mesons and charged particles [31], are compatible over the whole $p_{\mathrm{T}}$ range probed with the results in pp collisions scaled with the atomic mass number $A$ of the $\mathrm{Pb}$ nucleus. Moreover, the yields of $\mathrm{J} / \psi$ from $\mathrm{B}$ hadron decays as well as prompt $\mathrm{J} / \psi$ are found to be suppressed at low $p_{\mathrm{T}}$ at midrapidity in $\mathrm{MB} \mathrm{p}-\mathrm{Pb}$ collisions at $\sqrt{s_{\mathrm{NN}}}=5.02 \mathrm{TeV}$ [32], but the measurements of $\mathrm{B}$ hadron production cross sections at high $p_{\mathrm{T}}$ show no significant modification of the spectra compared to perturbative QCD calculations of pp collisions scaled with $A$. All of these results indicate that possible CNM effects are small compared to the current uncertainties of the measurements for open heavyflavor production at midrapidity at the LHC. However, at forward and backward rapidities, the measured $p_{\mathrm{T}}$ differential cross sections of D [33] and B mesons [34], and of muons originating from heavy-flavor hadron decays [35] in minimum bias $\mathrm{p}-\mathrm{Pb}$ collisions at $\sqrt{s_{\mathrm{NN}}}=5.02 \mathrm{TeV}$ demonstrate the presence of CNM effects and support shadowing as possible explanation. The forward and backward results set constraints on models that also aim at reproducing the midrapidity measurements. Accurate measurements in $\mathrm{pA}$ collisions provide important inputs for the parametrizations of the nuclear PDFs, which are currently suffering from large uncertainties $[18,19]$.

However, final-state effects may also play an important role in pA collisions. In particular, in those with large multiplicities of produced particles, as suggested by results from azimuthal anisotropy measurements through two-particle [36-42] and multi-particle correlations [43,44], modifications of the $p_{\mathrm{T}}$ distributions of identified hadrons with respect to the charged-particle multiplicity in the event [45,46], multiplicity dependence of strangeness production [47], and $\psi(2 \mathrm{~S})$ production [48-50]. Should such observations be linked to the creation of a small volume of hot medium in high-multiplicity pA collisions, the corresponding thermal radiation could lead to an enhanced dilepton production [51-53]. At RHIC energies, results on dilepton production at midrapidity in minimum bias d-Au collisions at $\sqrt{s_{\mathrm{NN}}}=200 \mathrm{GeV}[12,13]$ show no evidence of neither an additional source of lepton pairs, nor of nuclear modification of the charm and beauty production. At the LHC, where the density of final-state particles is larger, dilepton measurements in $\mathrm{p}-\mathrm{Pb}$ collisions can give more insight into the possible formation of a hot medium in small systems and CNM effects.

In this article, the first measurements of $\mathrm{e}^{+} \mathrm{e}^{-}$production in $\mathrm{pp}$ and $\mathrm{p}-\mathrm{Pb}$ collisions at $\sqrt{s_{\mathrm{NN}}}=5.02 \mathrm{TeV}$ at the LHC are presented. The results are obtained with the ALICE detector. The data are compared, in terms of the $m_{\mathrm{ee}}$ and $p_{\mathrm{T} \text {,ee }}$ distributions, to the sum of the expected sources of $\mathrm{e}^{+} \mathrm{e}^{-}$pairs from known hadron decays, the so-called hadronic cocktail. The spectra are shown after the application of fiducial requirements on single electrons $\left(\left|\eta_{\mathrm{e}}\right|<0.8\right.$ and $\left.0.2<p_{\mathrm{T}, \mathrm{e}}<10 \mathrm{GeV} / c\right)$ without an extrapolation to the full phase space. In addition, for the first time at LHC energies, a direct comparison between the dielectron cross section obtained in $\mathrm{pp}$ and $\mathrm{p}-\mathrm{Pb}$ collisions is possible since both data sets were recorded at the same $\sqrt{s_{\mathrm{NN}}}$. In particular, the analysis of the pp data resolves the model dependence on the expected $m_{\mathrm{ee}}$ and $p_{\mathrm{T}, \mathrm{ee}}$ distributions of correlated $\mathrm{e}^{+} \mathrm{e}^{-}$pairs from open heavy-flavor hadron decays in pp collisions, used as reference for the $\mathrm{p}-\mathrm{Pb}$ study. This allows for the research of possible modifications to the dielectron production in $\mathrm{p}-\mathrm{Pb}$ collisions due to CNM or additional final-state effects.

The article is organized as follows. The experimental setup and the used data samples are described in Sec. II. 
The analysis steps, including track selection criteria, electron identification, signal extraction and efficiency corrections, are described in Sec. III, together with the corresponding systematic uncertainties. The method to calculate the expected dielectron cross section from known hadron decays is explained in Sec. IV. In Sec. V, the results are presented, covering the charm and beauty cross section extracted in pp collisions, comparisons of the dielectron production in $\mathrm{pp}$ and $\mathrm{p}-\mathrm{Pb}$ collisions to the expectations from known hadron decays, and the resulting dielectron nuclear modification factors.

\section{THE ALICE DETECTOR AND DATA SAMPLES}

The ALICE detector and its performance are described in Refs. [1,54]. Electrons are measured in the ALICE central barrel covering the midrapidity range $|\eta|<0.9$. (Note that the term "electron" is used for both electrons and positrons throughout this paper.) The relevant subsystems used in the dielectron analysis are the inner tracking system (ITS) [55], the time projection chamber (TPC) [56], and the time-of-flight (TOF) [57] detector.

The innermost detector of the ALICE apparatus, closest to the nominal interaction point, is the ITS. It consists of six silicon tracking layers based on three different technologies. The two inner layers are silicon pixel detectors (SPD), the two middle layers are silicon drift detectors, and the two outer layers are silicon strip detectors. About half of the pp and $\mathrm{p}-\mathrm{Pb}$ data samples were recorded without the silicon drift detector information to reach maximal data acquisition rates. For this reason, even when available, the information from this detector is not used to have uniform detector conditions over the entire data sets. The main detector for particle identification (PID) and tracking is the TPC. This $500 \mathrm{~cm}$ long cylindrical detector, with an outer radius of $247 \mathrm{~cm}$, is located around the ITS. The TPC readout is based on multi-wire proportional chambers and provides up to 159 three-dimensional space points as well as the specific energy loss of the particle. The outermost detector used in this analysis is the TOF. It provides a time-of-flight measurement for particles from the interaction point to its active volume, at a radius of $370 \mathrm{~cm}$. The combined information from the ITS, TPC, and TOF is used to reconstruct the track of a charged particle using a Kalman-filter based algorithm [54].

The data used in this paper were recorded in collisions at $\sqrt{s_{\mathrm{NN}}}=5.02 \mathrm{TeV}$, with the p-Pb data taken in 2016, and the pp data taken in 2017. Due to the asymmetric beam energies in the $\mathrm{p}-\mathrm{Pb}$ configuration, $4 \mathrm{TeV}$ for the proton beam and $1.59 \mathrm{TeV}$ per nucleon for the $\mathrm{Pb}$ beam, the rapidity $(y)$ of the center-of-mass system is shifted by $\Delta y=0.465$ in the laboratory frame in the direction of the proton beam. For both collision systems, events were recorded when a coincident signal in the V0 detector system [58] was registered. The $\mathrm{V} 0$ detector consists of two segmented scintillators located at $+340 \mathrm{~cm}$ and $-70 \mathrm{~cm}$ along the beam axis from the nominal interaction point. Additional selections are applied to the recorded events. The background from beam-gas interactions and pileup events are rejected by using the correlations between the V0 detector and ITS signals. Only events with at least one track segment reconstructed in the ITS contribut-
TABLE I. The integrated luminosity $\left(\mathscr{L}_{\text {int }}\right)$ and the number of events $\left(N_{\mathrm{ev}}\right)$ after event selection criteria are applied for the pp and $\mathrm{p}-\mathrm{Pb}$ data samples.

\begin{tabular}{|c|c|c|}
\hline Data set & $\mathscr{L}_{\text {int }}$ & $N_{\mathrm{ev}}$ \\
\hline $\mathrm{pp}$ & $19.93 \pm 0.4 \mathrm{nb}^{-1}$ & $888 \times 10^{6}$ \\
\hline $\mathrm{p}-\mathrm{Pb}$ & $299 \pm 11 \mu \mathrm{b}^{-1}$ & $535 \times 10^{6}$ \\
\hline
\end{tabular}

ing to the vertex reconstruction with the SPD are used. To assure a uniform detector coverage at midrapidity, the vertex position along the beam direction is restricted to $\pm 10 \mathrm{~cm}$ with respect to the nominal interaction point. A summary of the number of events $N_{\text {ev }}$ passing the event selection criteria and the corresponding integrated luminosity $\mathscr{L}$ int is given in Table I. These requirements are fulfilled by $77 \%(75 \%)$ of the recorded events for the pp (p-Pb) data samples. The $\mathscr{L}_{\text {int }}$ is calculated as $\mathscr{L}_{\text {int }}=N_{\mathrm{MB}} / \sigma_{\mathrm{MB}}$, with the number of analyzed events after the vertex reconstruction efficiency correction $N_{\mathrm{MB}}$, and the minimum bias trigger cross section $\sigma_{\mathrm{MB}}$ measured via a van der Meer scan in the corresponding collision system $[59,60]$.

\section{DATA ANALYSIS}

\section{A. Track selection}

The same track selection criteria are applied in the analysis of the $\mathrm{pp}$ and $\mathrm{p}-\mathrm{Pb}$ data samples. Electron candidates are selected from charged tracks reconstructed in the ITS and TPC in the transverse-momentum range $0.2<p_{\mathrm{T}, \mathrm{e}}<10 \mathrm{GeV} / c$ and pseudorapidity range $\left|\eta_{\mathrm{e}}\right|<0.8$. The tracks are required to have at least 80 space points reconstructed in the TPC and at least three hits in the ITS assigned to them. The maximum $\chi^{2}$ per space point measured in the TPC (ITS) is required to be smaller than 4 (4.5). To reduce the contribution from secondary tracks, the distance-of-closest approach of the track to the reconstructed primary vertex is required to be smaller than $1 \mathrm{~cm}$ in the transverse plane to the colliding beams and smaller than $3 \mathrm{~cm}$ in the longitudinal direction. To further suppress the contribution of electrons from photon conversions in the detector material, only tracks with a hit in the first layer of the SPD and no ITS cluster shared with any other reconstructed track are used in the analysis.

\section{B. Electron identification}

Electrons are identified by measuring their specific energy loss $d E / d x$ in the TPC and their velocity with the TOF as a function of their momentum. The momentum is estimated from the curvature of the track measured in the ITS and TPC. The PID is based on the detector PID response $n\left(\sigma_{i}^{\text {Det }}\right)$. This is expressed as the deviation between the measured PID signal of the track in the detector (Det) and its expected most probable value for a given particle hypothesis $i$ at the measured track momentum. This deviation is normalized to the detector resolution $\sigma$. Electrons are selected over the whole investigated momentum range in the interval $\left|n\left(\sigma_{e}^{\mathrm{TPC}}\right)\right|<3$, while the charged pion $\left(\pi^{ \pm}\right)$contribution is suppressed by requiring 

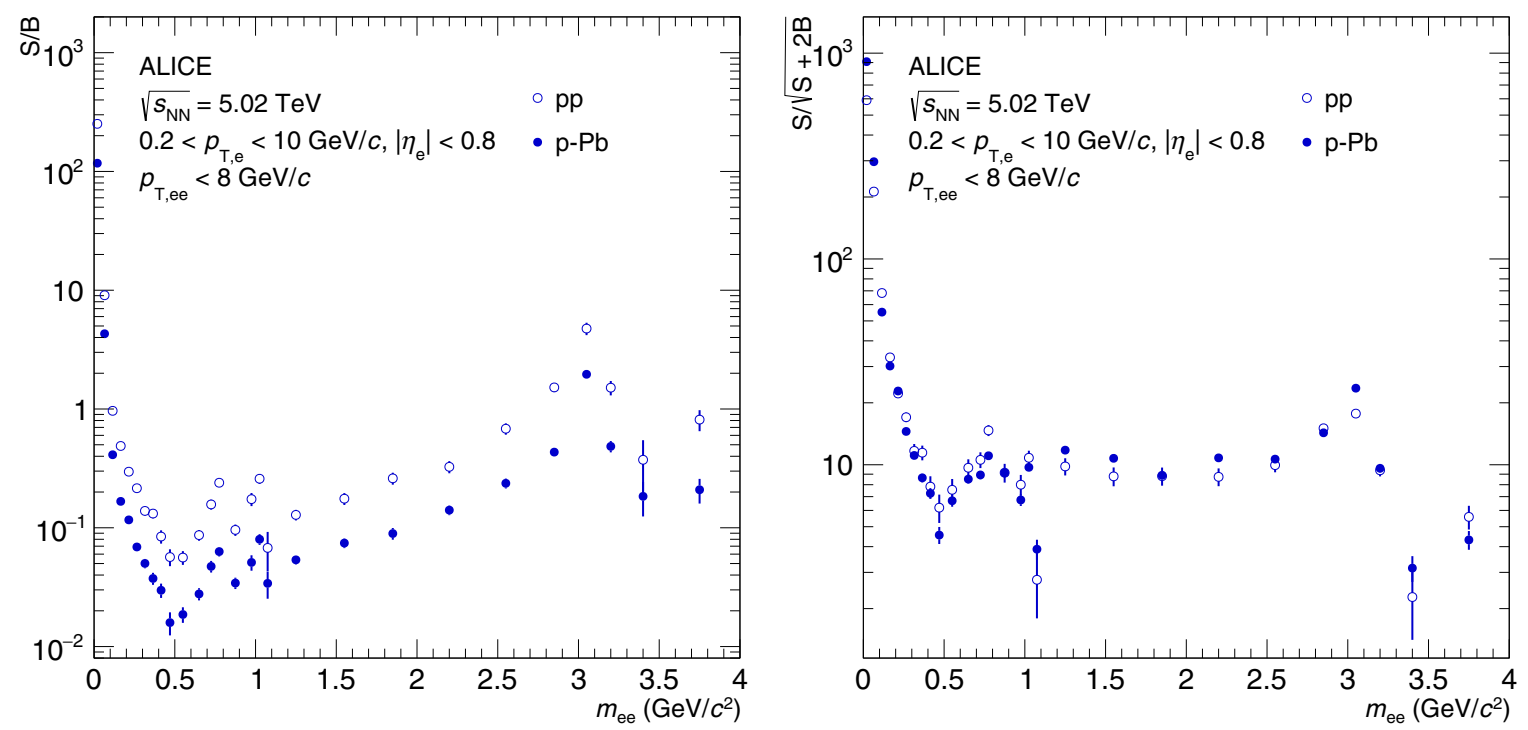

FIG. 1. Signal-to-background ratio (left) and statistical significance (right) of the dielectron measurements as a function of $m_{\mathrm{ee}}$ in pp and in $\mathrm{p}-\mathrm{Pb}$ collisions at $\sqrt{s_{\mathrm{NN}}}=5.02 \mathrm{TeV}$.

$n\left(\sigma_{\pi}^{\mathrm{TPC}}\right)>3.5$. Furthermore, the track must also fulfill at least one of the two following conditions:

(1) The track is outside the hadron bands in the TPC, defined by $\left|n\left(\sigma_{\mathrm{K}}^{\mathrm{TPC}}\right)\right|<3$ and $\left|n\left(\sigma_{\mathrm{p}}^{\mathrm{TPC}}\right)\right|<3$.

(2) The track has a valid hit in the TOF detector and falls within the range $\left|n\left(\sigma_{e}^{\mathrm{TOF}}\right)\right|<3$.

With this approach, the hadron contamination in the singleelectron candidate sample is less than $4 \%$ averaged over $p_{\mathrm{T}, \mathrm{e}}$. The largest hadron contamination, up to $9 \%$, is observed where kaons $\left(p_{\mathrm{T}} \approx 0.5 \mathrm{GeV} / c\right)$, protons $\left(p_{\mathrm{T}} \approx 1 \mathrm{GeV} / c\right)$ or charged pions $\left(p_{\mathrm{T}}>7 \mathrm{GeV} / c\right)$ have a similar $d E / d x$ as electrons in the TPC. The final hadron contamination in the dielectron signal is negligible, as pairs containing a misidentified hadron are further removed during the signal extraction.

\section{Signal extraction}

A statistical approach is used to extract the true signal pairs $(S)$ as a function of $m_{\mathrm{ee}}$ and $p_{\mathrm{T} \text {,ee, in which all electrons }}$ and positrons in an event are combined to create an oppositesign spectrum $(O S)$. The $O S$ contains not only signal, but also background $(B)$ from combinatorial pairs, as well as residual correlations from jets and conversions of correlated decay photons originating from the same particle. The background is estimated from the distribution of same-sign pairs $(S S)$ from the same event, as explained in Ref. [14]. The advantages of the same-sign technique, with respect to an event-mixing approach, are the intrinsic correct normalization of the $S S$ spectrum, and the inclusion of charge-symmetric background sources, e. g., electrons from fragmentation in jets. The signal is then extracted as $S=O S-R_{\text {acc }} \times S S$, where $R_{\text {acc }}$ is a correction factor needed to account for the different acceptance of opposite-sign and same-sign pairs. It is estimated using an event-mixing technique detailed in Ref. [14].

For pairs with $m_{\mathrm{ee}}<0.14 \mathrm{GeV} / c^{2}$, the angle $\varphi_{\mathrm{V}}$, which quantifies the orientation of the opening angle of the pairs relative to the magnetic field [14] and allows for the rejection of $\mathrm{e}^{+} \mathrm{e}^{-}$pairs from photon conversions, is required to be smaller than $2 \mathrm{rad}$. After applying this criterion, the remaining contribution from $\mathrm{e}^{+} \mathrm{e}^{-}$pairs from photon conversions in the detector material is less than $1.4 \%$.

The signal-to-background ratio $(S / B)$ and statistical significance $(S / \sqrt{S+2 B})$ are depicted in the left and right panels of Fig. 1, respectively, for the pp and $\mathrm{p}-\mathrm{Pb}$ samples. Despite a worse $S / B$ in $\mathrm{p}-\mathrm{Pb}$ collisions, mostly due to the larger particle multiplicity, the statistical significance of the measurement is similar in both collision systems.

\section{Efficiency correction}

The efficiency of the single-electron and pair selection is calculated with dedicated MC simulations. The simulated events are propagated through the ALICE detector using the GEANT 3 [61,62] transport code. The same strategy is used for the $\mathrm{pp}$ and $\mathrm{p}-\mathrm{Pb}$ analyses. Since the full kinematic range cannot be fully populated by pairs originating only from the same-mother particle (SM) or only from open heavyflavor hadron decays (HF), the final efficiency correction is estimated separately for each source. For SM pairs, pp and $\mathrm{p}-\mathrm{Pb}$ collisions are generated with the Monash2013 [63] tune of PYTHIA 8.1 [64] (denoted as PYTHIA 8 from now on) and with DPMJET [65], respectively. In the case of HF pairs, MC simulations of open heavy-flavor hadrons using PYTHIA 6 [66] are performed. In the $\mathrm{p}-\mathrm{Pb}$ case, heavy-flavor events are embedded into realistic $\mathrm{p}-\mathrm{Pb}$ collisions simulated with EPOS-LHC [67]. The efficiency as a function of $m_{\mathrm{ee}}$ and $p_{\mathrm{T}, \text { ee }}$ is calculated as

$$
\begin{aligned}
\epsilon_{\mathrm{ee}}\left(m_{\mathrm{ee}}, p_{\mathrm{T}, \mathrm{ee}}\right)= & w_{\mathrm{SM}} \times \epsilon_{\mathrm{SM} \rightarrow \mathrm{ee}}\left(m_{\mathrm{ee}}, p_{\mathrm{T}, \mathrm{ee}}\right) \\
& +w_{\mathrm{HF}} \times \epsilon_{\mathrm{HF} \rightarrow \mathrm{ee}}\left(m_{\mathrm{ee}}, p_{\mathrm{T}, \mathrm{ee}}\right) .
\end{aligned}
$$

The weights $w_{\mathrm{SM}}$ and $w_{\mathrm{HF}}$ represent the relative cross sections of the SM and HF sources, respectively. They are estimated with the expected dielectron cross section from known hadron decays, explained in Sec. IV. The average reconstruction 
TABLE II. Systematic uncertainties on the requirement of a hit in the first ITS layer, the ITS-TPC matching efficiency (ME), the allowed number of shared clusters in the ITS, the variation of the $\varphi_{\mathrm{V}}$ selection, and the tracking and PID variations in coarse $m_{\mathrm{ee}}$ intervals for the $\mathrm{p}-\mathrm{Pb}(\mathrm{pp})$ analysis. The uncertainties on the vertex reconstruction $(2 \%)$ and trigger $(2 \%)$ efficiencies in the pp analysis, as well as the uncertainty of the light- and heavy-flavor efficiency differences (3\%) in the $\mathrm{p}-\mathrm{Pb}$ analysis, are not listed. They are applied over the whole range of the measurement and included in the total uncertainty. The total systematic uncertainty is the quadratic sum of the single contributions assuming they are all uncorrelated.

\begin{tabular}{lccccc}
\hline \hline$m_{\mathrm{ee}}\left(\mathrm{GeV} / c^{2}\right)$ & 1st ITS layer & ITS-TPC ME & Shared ITS cls. & $\varphi_{\mathrm{V}}$ & Tracking \& PID \\
\hline$<0.14$ & $2(1) \%$ & $2(2) \%$ & $2(1) \%$ & $2(1) \%$ & $10(6) \%$ \\
$0.14-1.1$ & $2(1) \%$ & $2(2) \%$ & $2(0) \%$ & - & $2(2) \%$ \\
$1.1-2.7$ & $2(2) \%$ & $2(3) \%$ & $0(0) \%$ & - & $2(2) \%$ \\
$2.7-3.5$ & $2(2) \%$ & $2(4) \%$ & $0(0) \%$ & - & $5(4) \%$ \\
\hline \hline
\end{tabular}

efficiency of a signal $\mathrm{e}^{+} \mathrm{e}^{-}$pair is very similar in $\mathrm{pp}$ and $\mathrm{p}-\mathrm{Pb}$ collisions and ranges from $25 \%$ to $30 \%$.

The corrected differential dielectron cross section is calculated as

$$
\frac{d^{2} \sigma_{\mathrm{ee}}}{d m_{\mathrm{ee}} d p_{\mathrm{T}, \mathrm{ee}}}=\frac{1}{\mathscr{L}_{\mathrm{int}}} \frac{1}{\Delta m_{\mathrm{ee}}} \frac{1}{\Delta p_{\mathrm{T}, \mathrm{ee}}} \frac{S\left(m_{\mathrm{ee}}, p_{\mathrm{T}, \mathrm{ee}}\right)}{\epsilon_{\mathrm{ee}}\left(m_{\mathrm{ee}}, p_{\mathrm{T}, \mathrm{ee}}\right)},
$$

with $\Delta m_{\mathrm{ee}}$ and $\Delta p_{\mathrm{T}, \mathrm{ee}}$ being the width of the $m_{\mathrm{ee}}$ and $p_{\mathrm{T}, \mathrm{ee}}$ intervals, respectively, and $\mathscr{L}_{\text {int }}$ the integrated luminosity. In pp collisions, the spectra are corrected for the vertex reconstruction efficiency and for the efficiency of the minimum bias trigger to select inelastic events with an $\mathrm{e}^{+} \mathrm{e}^{-}$pair, which are found to be $96 \%$ and $98 \%$, respectively. In $\mathrm{p}-\mathrm{Pb}$ collisions both efficiencies are unity.

\section{E. Systematic uncertainties}

Different sources of systematic uncertainties are taken into account. On the single track level the effects of the required hit in the first ITS layer, the ITS-TPC matching efficiency, and the selection of tracks without shared clusters are studied. These uncertainties are calculated as a function of $m_{\mathrm{ee}}$ and $p_{\mathrm{T}, \text { ee }}$. Effects from the track and PID selection as well as the requirement on $\varphi_{\mathrm{V}}$ are estimated on the pair level. For these uncertainties negligible $p_{\mathrm{T}, \text { ee }}$ dependence is found and they are applied only as a function of $m_{\mathrm{ee}}$. To suppress statistical fluctuations, that could influence the estimated systematic uncertainties, they are evaluated in both analyses in wide mass intervals. The resulting systematic uncertainties from the different sources are summarized in Table II for the $\mathrm{p}-\mathrm{Pb}$ and pp analyses.

The systematic uncertainties that arise from the limited knowledge of the matching efficiency of the track segments reconstructed in the ITS and the TPC, and from the requirement of a hit in the innermost ITS layer, are determined with a two-step procedure. First, on the single-track level, the efficiencies of these two track selection criteria are estimated for charged pions in data and in MC as a function of $p_{\mathrm{T}}$. Second, the observed difference is taken as input to a toy MC simulation, which generates particles in the full $m_{\mathrm{ee}}$ and $p_{\mathrm{T}, \mathrm{ee}}$ phase space decaying them into $\mathrm{e}^{+} \mathrm{e}^{-}$pairs and applying the fiducial selection. The final systematic uncertainty at the pair level is then calculated as the sum of the uncertainties of the decay products, corresponding to the input.
The systematic uncertainty from the requirement of no shared clusters in the ITS is evaluated by varying the maximum number of allowed shared ITS clusters for the selected electron candidates. This provides a test of the understanding of the background since it not only probes different singleelectron efficiencies but also different $S / B$ ratios. When no requirement is applied the $S / B$ decreases by a factor two, which is due to the increased contribution of electrons from photon conversions in the detector material in the selected electron sample. The resulting dielectron spectra are compared after the efficiency correction. The maximum deviation of the variations that are considered statistically significant according to the Barlow criterion [68] is used to assign the systematic uncertainty.

Similarly, the uncertainty from the remaining singleelectron selection criteria is determined by varying them simultaneously within reasonable values. By changing the selection criteria for the tracks in the ITS and the hadron rejection criteria, the evaluated systematic uncertainties are sensitive to estimations of the background as well as a possible bias due to the hadron contamination in the electron sample. The systematic uncertainty is calculated as the root mean square of the variation of the final data points. Finally, a possible bias due to the efficiency correction of the $\varphi_{\mathrm{V}}$ selection is estimated. For this purpose, the maximum $\varphi_{\mathrm{V}}$ requirement for $\mathrm{e}^{+} \mathrm{e}^{-}$pairs with $m_{\mathrm{ee}}<0.14 \mathrm{GeV} / c^{2}$ is varied around its default value from 1.5 to $2.7 \mathrm{rad}$.

Two additional sources of uncertainty are taken into account for the $\mathrm{pp}$ analysis, namely the correction for the primary vertex reconstruction efficiency and the trigger efficiency. Both are evaluated to be $2 \%$ based on MC simulations. A priori, the reconstruction efficiency of an $\mathrm{e}^{+} \mathrm{e}^{-}$pair at a given $m_{\mathrm{ee}}$ and $p_{\mathrm{T} \text {,ee }}$ should not depend on its source. However, in the $\mathrm{p}-\mathrm{Pb}$ analysis, a difference in the efficiencies of $\mathrm{e}^{+} \mathrm{e}^{-}$pairs originating from either light-flavor decays or heavy-flavor decays is observed. Therefore, an additional uncertainty of $3 \%$ is assigned to cover a possible bias in the spectra. The total systematic uncertainty is calculated as the quadratic sum of the individual contributions assuming they are all uncorrelated. The total uncertainty varies between $11 \%$ and $4 \%$, being equal to $5 \%$ in most of the $m_{\mathrm{ee}}$ range. The uncertainties are partially correlated between different $m_{\mathrm{ee}}$ intervals. 


\section{COCKTAIL OF KNOWN HADRON DECAYS}

The measured dielectron spectra in $\mathrm{pp}$ and $\mathrm{p}-\mathrm{Pb}$ collisions are compared to a hadronic cocktail, which represents the sum of the expected contributions of dielectrons from known hadron decays, after the fiducial selection criteria on single electrons are employed. A fast MC simulation of the ALICE central barrel is performed, including realistic momentum and angular resolutions as well as Bremsstrahlung effects, which are applied to the decay electrons as a function of $p_{\mathrm{T}, \mathrm{e}}$, azimuthal angle $\left(\varphi_{\mathrm{e}}\right)$ and $\eta_{\mathrm{e}}$ [69].

The Dalitz and dielectron decays of light neutral mesons are simulated with the phenomenological event generator EXODUS [70], following the approach described in Ref. [14]. The $p_{\mathrm{T}}$ spectra of light neutral mesons measured at midrapidity in pp collisions at different center-of-mass energies and in $\mathrm{p}-\mathrm{Pb}$ collisions at $\sqrt{s_{\mathrm{NN}}}=5.02 \mathrm{TeV}$ are parametrized and taken as input to the calculations. Since the measured $p_{\mathrm{T}}$ distributions of $\pi^{ \pm}$mesons extend to lower $p_{\mathrm{T}}$, they are used to determine the $\pi^{0}$ input parametrizations. The $p_{\mathrm{T}}$ spectra of $\pi^{ \pm}$mesons measured by ALICE in pp and $\mathrm{p}-\mathrm{Pb}$ collisions at $\sqrt{s_{\mathrm{NN}}}=5.02 \mathrm{TeV}[71,72]$ are first parametrized with a modified Hagedorn function [73]. A $p_{\mathrm{T}}$-dependent scaling factor is then applied to the $\pi^{ \pm}$parametrization to account for the difference between $\pi^{0}$ and $\pi^{ \pm}$due to isospin-violating decays, mainly of the $\eta$ mesons. This factor is estimated using an effective model that describes measured hadron spectra at low $p_{\mathrm{T}}$ and includes strong and electromagnetic decays. The measured $p_{\mathrm{T}}$ spectra of $\phi$ mesons in $\mathrm{pp}$ and $\mathrm{p}-\mathrm{Pb}$ collisions at $\sqrt{s_{\mathrm{NN}}}=5.02 \mathrm{TeV}[74,75]$ are fitted to obtain the $\phi$ input parametrizations. The $p_{\mathrm{T}}$ spectra of the other light mesons, $\eta, \eta^{\prime}, \rho$, and $\omega$ are derived from the $\pi^{ \pm}$spectrum. The $p_{\mathrm{T}}$ spectrum of the $\eta$ meson is estimated from a common fit to the ratios of the $\eta$ to $\pi^{0} p_{\mathrm{T}}$ spectra in pp collisions at $\sqrt{s}=7 \mathrm{TeV}$ [76], $8 \mathrm{TeV}$ [77], and in $\mathrm{p}-\mathrm{Pb}$ collisions at $\sqrt{s_{\mathrm{NN}}}=5.02 \mathrm{TeV}$ [78] measured by ALICE as well measurements by CERES/TAPS in p-Au and $\mathrm{p}-$ Be collisions at $\sqrt{s_{\mathrm{NN}}}=29.1 \mathrm{GeV}$ which extend to lower $p_{\mathrm{T}}\left(p_{\mathrm{T}}<2 \mathrm{GeV} / c\right)$ [79]. The $p_{\mathrm{T}}$ distributions of $\omega$ and $\rho$ are obtained from the respective ratios to the $\pi^{ \pm} p_{\mathrm{T}}$ distributions in simulated pp collisions at $\sqrt{s}=5.02 \mathrm{TeV}$ with PYTHIA 8. The $\eta / \pi^{0}, \rho / \pi^{ \pm}$and $\omega / \pi^{ \pm}$ratios as a function of $p_{\mathrm{T}}$ are assumed to be independent of the $\mathrm{pA}$ or pp collision system and of the energy, as suggested by the measurements [76-79]. Therefore, common parametrizations of these ratios are used for the $\mathrm{pp}$ and $\mathrm{p}-\mathrm{Pb}$ cocktails. Finally, the $\eta^{\prime}$ meson is generated assuming $m_{\mathrm{T}}$ scaling [80-82], implying that the spectra of all light mesons as a function of $m_{\mathrm{T}}=\sqrt{m_{0}^{2}+p_{\mathrm{T}}{ }^{2}}$, where $m_{0}$ is the pole mass of the considered mesons, follow the same shape and only differ by a normalization factor. All contributions from the decays of light-flavor hadrons as a function of $m_{\mathrm{ee}}$ are shown in Fig. 2. To estimate the $\mathrm{J} / \psi$ contribution, the measured $\mathrm{J} / \psi p_{\mathrm{T}}$ spectra in $\mathrm{pp}$ and $\mathrm{p}-\mathrm{Pb}$ collisions at $\sqrt{s_{\mathrm{NN}}}=5.02 \mathrm{TeV}[83,84]$ are parametrized and used as inputs for the simulations. The $\mathrm{J} / \psi$ mesons are decayed using PHOTOS [85] via the dielectron channel, which also includes the full QED radiative channels.

The contributions of correlated semileptonic decays of open charm and beauty hadrons are calculated with two differ-

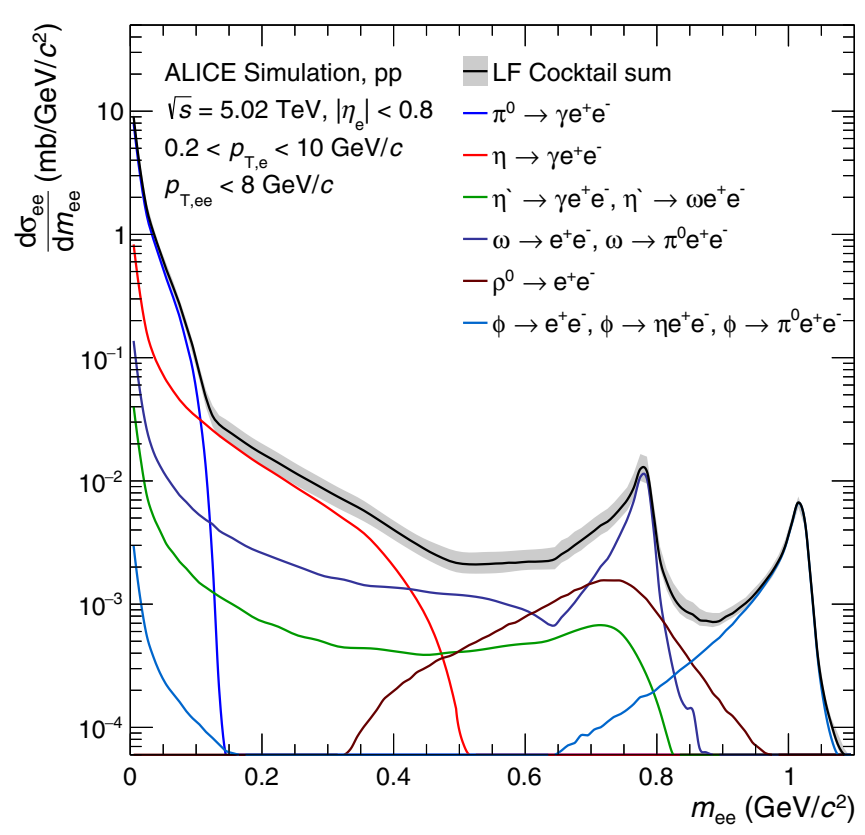

FIG. 2. Expected cross section for dielectron production from light-flavor hadron decays in pp collisions at $\sqrt{s_{\mathrm{NN}}}=5.02 \mathrm{TeV}$ as a function of $m_{\mathrm{ee}}$. The sum of the single light-flavor (LF) contributions is shown (solid black line) with its uncertainties (gray band).

ent MC event generators. They are identical to the ones used in the dielectron analyses performed by ALICE in pp collisions at $\sqrt{s}=7 \mathrm{TeV}$ [14] and $\sqrt{s}=13 \mathrm{TeV}$ [15]: PYTHIA 6.4 [66] with the Perugia2011 tune [86] and the next-to-leading order event generator POWHEG [87-90] with PYTHIA 6 to evolve the parton shower. Only the shapes of the expected $m_{\mathrm{ee}}$ and $p_{\mathrm{T}, \text { ee }}$ dielectron spectra are estimated with the MC event generators. The absolute normalization is obtained from a fit of the measured dielectron cross sections in pp collisions at $\sqrt{s}=5.02 \mathrm{TeV}$, as shown in Sec. V A. For $\mathrm{p}-\mathrm{Pb}$ collisions, the $c \bar{c}$ and $b \bar{b}$ cross sections, extracted in pp collisions, are scaled with the atomic mass number $A$ of the $\mathrm{Pb}$ nucleus (208). This approach neglects any cold nuclear matter effects, which will be discussed in Sec. V B.

The following sources of systematic uncertainties are taken into account: the input parametrizations of the measured $\pi^{ \pm}$, $\phi$ and $\mathrm{J} / \psi p_{\mathrm{T}}$ spectra and $\eta / \pi^{0}$ ratios, the scaling factor applied to the $\pi^{ \pm}$parametrizations, the $m_{\mathrm{T}}$ scaling parameters, and the different decay branching ratios. The uncertainty of the $\pi^{ \pm}$scaling factor is estimated from variations of the model parameters. For the $\rho$ and $\omega$ mesons, the uncertainty of the $\omega / \pi^{0}$ and $\rho / \pi^{0}$ ratios are estimated by comparing the measured and simulated ratios in pp collisions at $\sqrt{s}=7 \mathrm{TeV}$ [91] and $\sqrt{s}=2.76 \mathrm{TeV}$ [92], respectively. The total uncertainties of the pp and $\mathrm{p}-\mathrm{Pb}$ cocktails vary from $5 \%$ to $20 \%$ depending on the $m_{\mathrm{ee}}$ and $p_{\mathrm{T}, \mathrm{ee}}$ interval.

\section{RESULTS}

The dielectron cross sections in $\mathrm{pp}$ and $\mathrm{p}-\mathrm{Pb}$ collisions as well as the nuclear modification factor are presented differentially as a function of $m_{\mathrm{ee}}$ for $p_{\mathrm{T} \text {,ee }}<8 \mathrm{GeV} / c$ and 

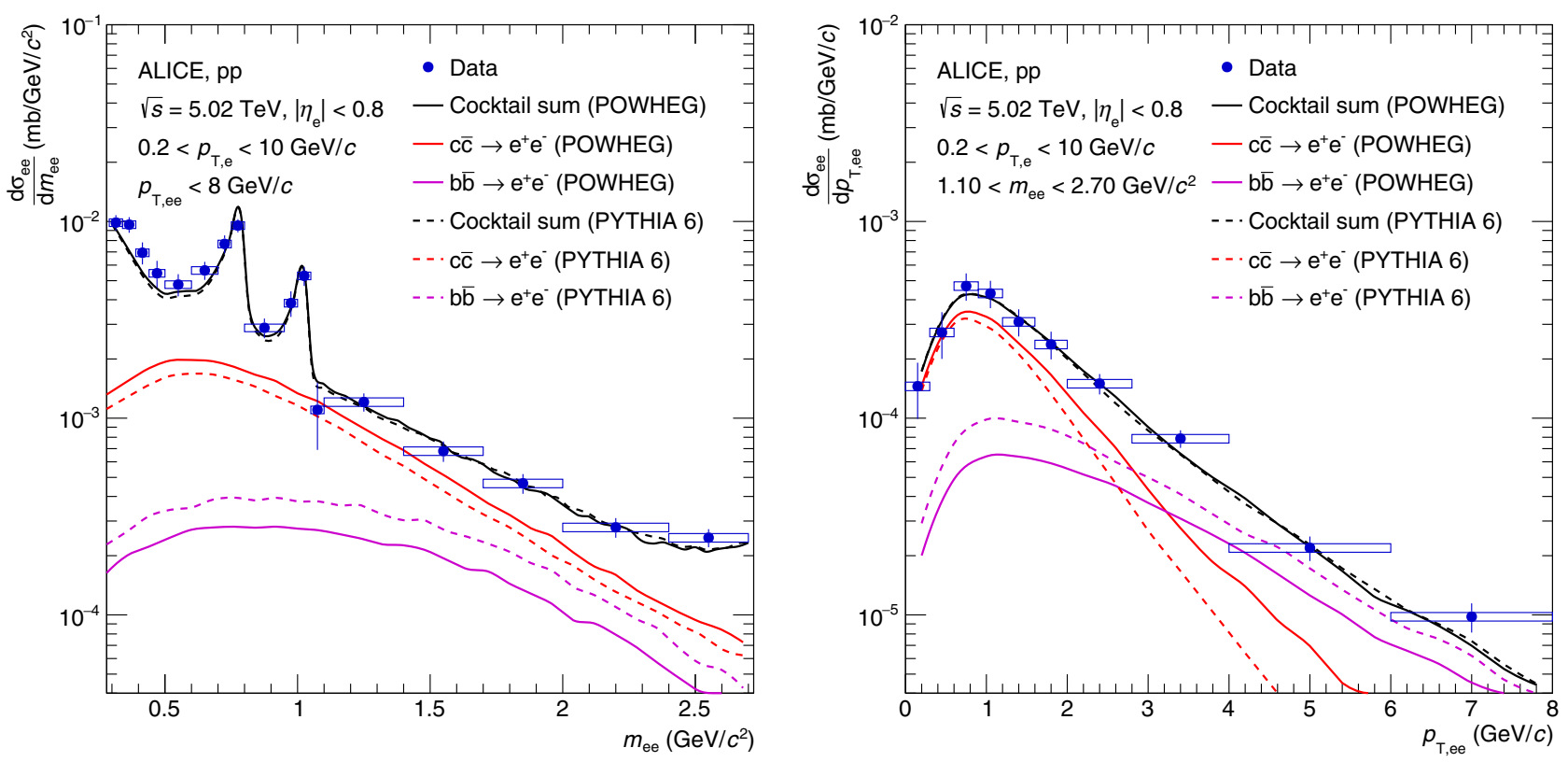

FIG. 3. Projections of the heavy-flavor dielectron fits as a function of $m_{\mathrm{ee}}$ (left) and $p_{\mathrm{T}, \mathrm{ee}}$ (right) using POWHEG (solid black line) and PYTHIA 6 (dashed gray line) as event generators. The colored lines show the charm (red) and beauty (magenta) contributions for both event generators after the fit.

as a function of $p_{\mathrm{T}, \mathrm{ee}}$ in two different mass regions, the low-mass region (LMR), $0.5<m_{\mathrm{ee}}<1.1 \mathrm{GeV} / c^{2}$, and the intermediate-mass region (IMR), $1.1<m_{\mathrm{ee}}<2.7 \mathrm{GeV} / c^{2}$.

\section{A. Heavy-flavor cross sections in pp collisions}

The differential $\mathrm{e}^{+} \mathrm{e}^{-}$production cross sections $\mathrm{d} \sigma_{\mathrm{ee}} / \mathrm{d} m_{\mathrm{ee}}$ and $\mathrm{d} \sigma_{\mathrm{ee}} / \mathrm{d} p_{\mathrm{T}, \mathrm{ee}}$ in pp collisions, measured in the IMR and in the range $p_{\mathrm{T} \text {,ee }}<8 \mathrm{GeV} / c$ at $\sqrt{s}=5.02 \mathrm{TeV}$ are presented in Fig. 3. The data in the IMR are fitted in $m_{\mathrm{ee}}$ and $p_{\text {T,ee }}$ with PYTHIA 6 and POWHEG templates of opencharm (red) and open-beauty (magenta) production, keeping the light-flavor and $\mathrm{J} / \psi$ contributions fixed. In this mass range, most of the $\mathrm{e}^{+} \mathrm{e}^{-}$pairs originate from open heavyflavor hadron decays. The $\chi^{2} /$ ndf between the data and the cocktail sum is $110.9 / 123$ for the POWHEG cocktail and 113.4/123 for the PYTHIA 6 cocktail. Both calculations are able to reproduce the measured spectra well over the full kinematic range probed, however the full cocktail obtained with POWHEG leads to a slightly better description of the data at low $m_{\mathrm{ee}}$ around $m_{\mathrm{ee}}=0.5 \mathrm{GeV} / c^{2}$. The resulting cross sections are listed in Table III. The systematic uncertainties originating from the data were determined by repeating the fit after moving the data points coherently up- and downward by their systematic uncertainties. Additional uncertainties on the effective beauty- and charm-to-electron branching ratios, arising from the semileptonic decay branching ratios of open heavy-flavor hadrons and the fragmentation functions of charm (beauty) quarks, amounting to $22 \%$ and $6 \%$ for the charm and beauty cross sections, respectively, are also listed in the table. All uncertainties are fully correlated between the two generators, which differ only in the implementation of the heavy-quark production mechanisms. In both calculations, the hadronization of the c- and b-quarks, and the decays of the open heavy-flavor hadrons, are performed using PYTHIA 6. For the following results, only calculations where the heavyflavor contribution is evaluated with POWHEG are presented, since the cocktail using POWHEG and fitted to the data in the IMR can slightly better describe the measured dielectron cross sections over the full $m_{\mathrm{ee}}$ and $p_{\mathrm{T}, \mathrm{ee}}$ range in pp collisions at $\sqrt{s}=5.02 \mathrm{TeV}$.

A compilation of the measured $d \sigma_{\mathrm{c} \bar{c}} /\left.d y\right|_{y=0}$ (left) and $d \sigma_{\mathrm{b} \overline{\mathrm{b}}} /\left.d y\right|_{y=0}$ (right) in pp collisions at LHC energies is shown in Fig. 4 as a function of $\sqrt{s}$. The difference in the cross sections obtained with the two MC event generators in the present analysis at $\sqrt{s}=5.02 \mathrm{TeV}$ is comparable with the results of previous observations at $\sqrt{s}=7$ [14] and $13 \mathrm{TeV}$ [15] performed with the same models. This reflects the sensitivity

TABLE III. Heavy-flavor cross sections extracted via double differential fits in $m_{\mathrm{ee}}$ and $p_{\mathrm{T}, \mathrm{ee}}$ to the measured dielectron spectra in pp collisions at $\sqrt{s}=5.02 \mathrm{TeV}$ using PYTHIA 6 and POWHEG. The statistical (stat.) and systematic (syst.) uncertainties on the data are quoted together with the $22 \%(6 \%)$ uncertainty on the branching ratio (BR) of the semileptonic decays of the open heavy-flavor hadrons and the fragmentation functions of charm (beauty) quarks.

\begin{tabular}{lcc}
\hline & PYTHIA & POWHEG \\
\hline$d \sigma_{\overline{\mathrm{c}} \mathrm{c}} /\left.d y\right|_{y=0}$ & $524 \pm 61$ (stat.) \pm 26 (syst.) $\pm 115(\mathrm{BR}) \mu \mathrm{b}$ & $756 \pm 80$ (stat.) \pm 38 (syst.) $\pm 166(\mathrm{BR}) \mu \mathrm{b}$ \\
$d \sigma_{\mathrm{b} \overline{\mathrm{b}}} /\left.d y\right|_{y=0}$ & $34 \pm 4$ (stat.) \pm 2 (syst.) $\pm 2(\mathrm{BR}) \mu \mathrm{b}$ & $28 \pm 5$ (stat.) \pm 1 (syst.) $\pm 2(\mathrm{BR}) \mu \mathrm{b}$ \\
\hline \hline
\end{tabular}



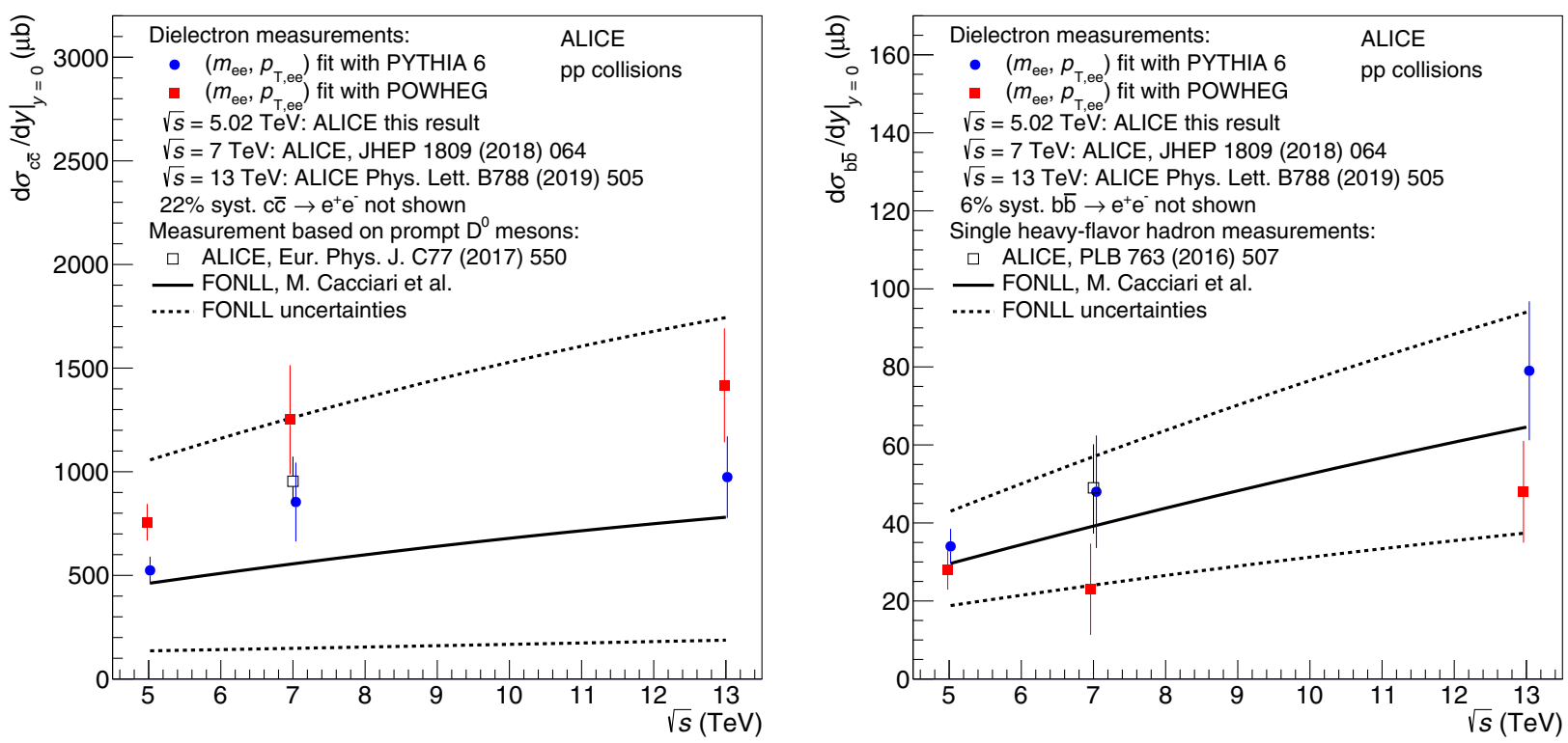

FIG. 4. Cross sections at midrapidity for $c \bar{c}$ (left) and $b \bar{b}$ (right) as a function of $\sqrt{s}$ in pp collisions. The colored markers represent the measured midrapidity cross sections at $\sqrt{s}=5.02$, 7, and $13 \mathrm{TeV}$ which are derived using either PYTHIA 6 (blue circles) or POWHEG (red squares) simulations. The systematic and statistical uncertainty of the data points are summed in quadrature and represented by vertical bars. The measurements are compared with FONLL calculations (black solid line), with model uncertainties (dashed lines), and to single heavy-flavor hadron measurements (open markers). The referenced cic cross section at $\sqrt{s}=7 \mathrm{TeV}$ was obtained from a measurement of prompt $\mathrm{D}^{0}$ meson production with $p_{\mathrm{T}}>0 \mathrm{GeV} / c$ and $|y|<0.5$ using the fragmentation fraction $\mathrm{f}\left(\mathrm{c} \rightarrow \mathrm{D}^{0}\right)=0.542 \pm 0.024$ from $\mathrm{e}^{+} \mathrm{e}^{-} \mathrm{LEP}$ data [93].

of the dielectron measurement to the implementation of the heavy-quark production mechanisms, in particular to the initial correlation of charm quarks, which is not accessible with conventional measurements of single open-charm hadrons and their decay products. Nevertheless, the cross sections measured using POWHEG or PYTHIA are all in agreement, within the current precision, with results from single heavy-

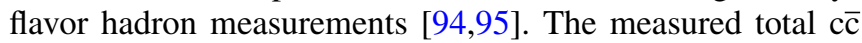
production cross section in pp collisions at $\sqrt{s}=7 \mathrm{TeV}$ was obtained from a measurement of prompt $\mathrm{D}^{0}$ meson production with $p_{\mathrm{T}}>0 \mathrm{GeV} / c$ and $|y|<0.5$ using the fragmentation fraction $\mathrm{f}\left(\mathrm{c} \rightarrow \mathrm{D}^{0}\right)=0.542 \pm 0.024$ from $\mathrm{e}^{+} \mathrm{e}^{-}$LEP data [93]. Recent measurements of $\mathrm{f}\left(\mathrm{c} \rightarrow \mathrm{D}^{0}\right)$ suggest that this value is smaller in pp collisions at the LHC [96], which would result in a larger cross section of charm production than assumed in Ref. [95]. FONLL calculations [16] are able to reproduce the measurements within the model uncertainties that are dominated by scale uncertainties, but also include PDF and mass uncertainties. The slope of the center-of-mass energy dependence of the cross sections is described by the calculations. The measured charm production cross sections are however on the upper edge of the large systematic uncertainties of the theory calculations for all three measurements.

\section{B. Dielectron production in $\mathrm{pp}$ and $\mathrm{p}-\mathrm{Pb}$ collisions}

The $m_{\mathrm{ee}}$-differential production cross sections of $\mathrm{e}^{+} \mathrm{e}^{-}$ pairs measured in $\mathrm{pp}$ and $\mathrm{p}-\mathrm{Pb}$ collisions at $\sqrt{s_{\mathrm{NN}}}=5.02 \mathrm{TeV}$ are compared to the expected dielectrons from known hadron decays in Fig. 5. The light-flavor contributions, summarized as "Light flavor" for readability, are based on measurements in $\mathrm{pp}$ and $\mathrm{p}-\mathrm{Pb}$ collisions as explained in detail in Sec. IV. The correlated pairs from heavy-flavor hadron decays are calculated with POWHEG. Their contributions are normalized to the $d \sigma_{\mathrm{c} \overline{\mathrm{c}}} /\left.d y\right|_{y=0}$ and the $d \sigma_{\mathrm{b} \overline{\mathrm{b}}} /\left.d y\right|_{y=0}$ in pp collisions obtained from the fit to the pp data, as discussed in the previous section. For $\mathrm{p}-\mathrm{Pb}$ collisions, the heavy-flavor contributions are further scaled with the atomic mass number of the $\mathrm{Pb}$ nucleus. This assumes that the production of heavy-flavor quarks in $\mathrm{p}-\mathrm{Pb}$ collisions scales with the number of binary nucleon-nucleon collisions. The total systematic uncertainty of the cocktails is indicated by the gray band. The pp cocktail uncertainty in the IMR is zero by construction since the heavy-flavor contribution is directly fitted to the measured spectrum in $\mathrm{pp}$ collisions. The systematic uncertainties of the heavy-flavor contribution in the $\mathrm{p}-\mathrm{Pb}$ cocktail originate from the statistical and systematic uncertainties of the extracted production cross sections in the pp analysis listed in Table III. Since the cross section is based on the measurement of final state $\mathrm{e}^{+} \mathrm{e}^{-}$pairs, the uncertainties related to branching ratios of the semileptonic decays of open heavy-flavor hadrons and the fragmentation functions of charm and beauty quarks can be omitted, under the assumption that these do not change from $\mathrm{pp}$ to $\mathrm{p}-\mathrm{Pb}$ collisions. This is confirmed by the latest measurements of open heavy-flavor hadrons in $\mathrm{pp}$ and $\mathrm{p}-\mathrm{Pb}$ collisions at $\sqrt{s_{\mathrm{NN}}}=5.02 \mathrm{TeV}$ by ALICE [96]. The bottom panels in Fig. 5 show the ratios of the data to the cocktail. The data are described by the hadronic cocktails over the whole mass range $\left(m_{\mathrm{ee}}<3.5 \mathrm{GeV} / c^{2}\right)$ in both $\mathrm{pp}$ and $\mathrm{p}-\mathrm{Pb}$ collisions, within the systematic and statistical 

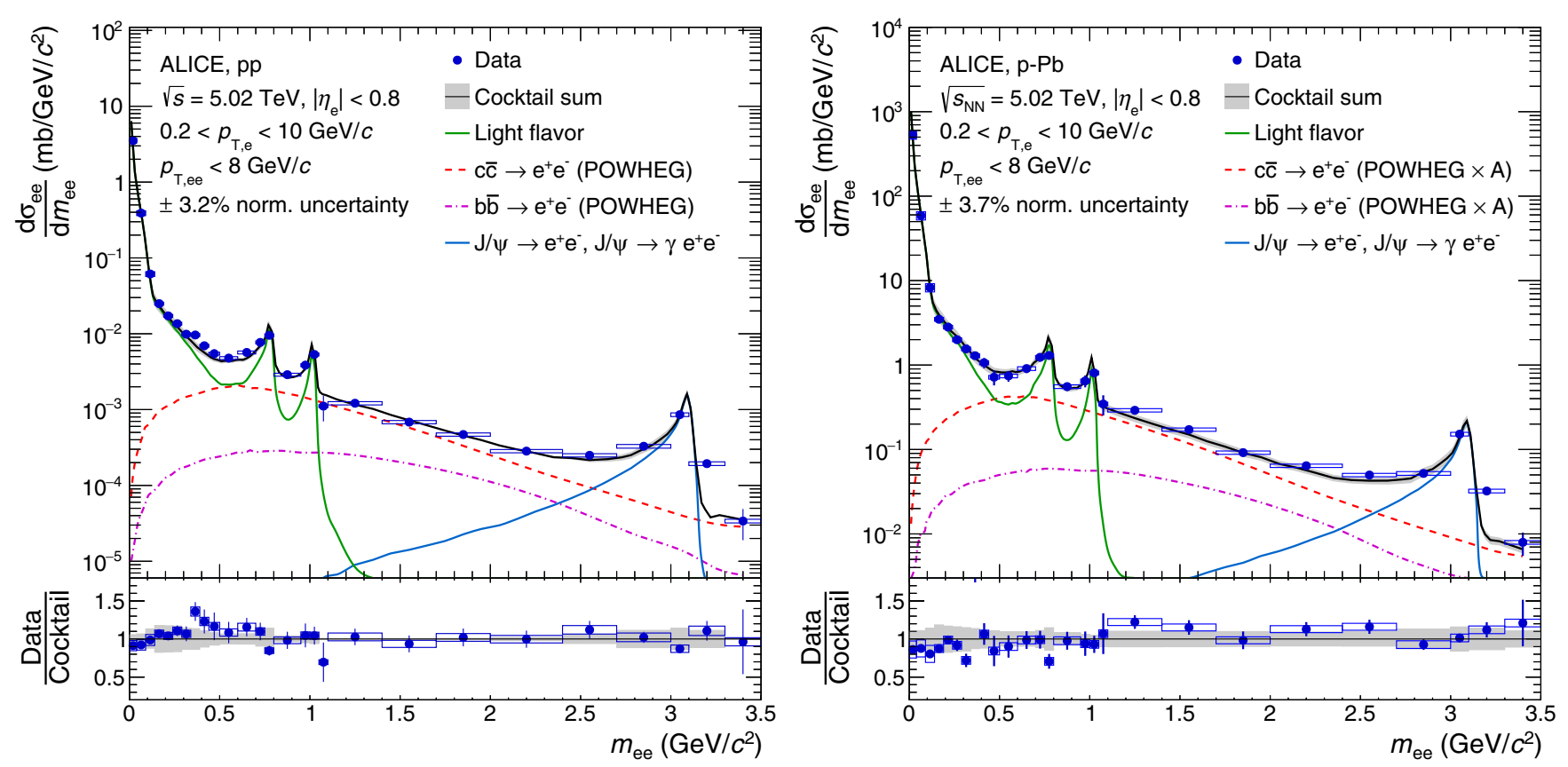

FIG. 5. Differential $\mathrm{e}^{+} \mathrm{e}^{-}$cross section as a function of $m_{\mathrm{ee}}$ measured in pp (left) and $\mathrm{p}-\mathrm{Pb}$ (right) collisions at $\sqrt{s_{\mathrm{NN}}}=5.02 \mathrm{TeV}$. The data are compared to the hadronic cocktail, where the heavy-flavor contributions are fitted to the pp spectrum in the intermediate-mass region, and for $\mathrm{p}-\mathrm{Pb}$ collisions scaled with the atomic mass number of the $\mathrm{Pb}$ nucleus $A=208$. The gray band represents the total uncertainty on the hadronic cocktail.

uncertainties. As seen in previous measurements in pp collisions $[14,15]$, the heavy-flavor contribution dominates the spectrum for $m_{\mathrm{ee}}>0.8 \mathrm{GeV} / c^{2}$. In $\mathrm{p}-\mathrm{Pb}$ collisions, the heavy-flavor contribution to the hadronic cocktail does not include any modification beyond scaling with binary nucleonnucleon collisions with respect to the pp cocktail. No significant deviation of the data from the vacuum expectation of the heavy-flavor contributions can be observed in the mass spectrum. This suggests that the CNM effects are small compared to the current uncertainties of the measurements, as observed by other open heavy-flavor measurements at the LHC at midrapidity [28], or compensated by an additional source of dielectrons in $\mathrm{p}-\mathrm{Pb}$ collisions compared with pp collisions, possibly related to the formation of a hot medium in such collisions.

The $p_{\mathrm{T}, \text { ee }}$ spectra for $\mathrm{pp}$ and $\mathrm{p}-\mathrm{Pb}$ collisions in the LMR and IMR are compared to the hadronic cocktail in Figs. 6 and 7, respectively. In the LMR, the hadronic cocktails in $\mathrm{pp}$ and $\mathrm{p}-\mathrm{Pb}$ collisions are both composed of $\mathrm{e}^{+} \mathrm{e}^{-}$pairs from light-flavor, open-charm, and open-beauty hadron decays. Most of the pairs in this mass interval are produced from the decays of light-flavor hadrons, whose production at low $p_{\mathrm{T}}$ does not scale with $A$ in $\mathrm{p}-\mathrm{Pb}$ collisions. Therefore, the relative expected contribution of dielectrons from lightflavor hadron decays is smaller in $\mathrm{p}-\mathrm{Pb}$ collisions compared with pp collisions at the same $\sqrt{s_{\mathrm{NN}}}$. In $\mathrm{p}-\mathrm{Pb}$ collisions, the open-charm hadron decays are expected to contribute significantly to the $\mathrm{e}^{+} \mathrm{e}^{-}$cross section for $p_{\mathrm{T} \text {,ee }}<1 \mathrm{GeV} / c$. The open-beauty contribution only plays a significant role for $p_{\mathrm{T}, \text { ee }}>4 \mathrm{GeV} / c$ in both collision systems. In the IMR, correlated $\mathrm{e}^{+} \mathrm{e}^{-}$pairs from open-charm hadron decays are the dominant dielectron source for $p_{\mathrm{T} \text {,ee }}<2.5 \mathrm{GeV} / c$ in $\mathrm{pp}$ as well as in $\mathrm{p}-\mathrm{Pb}$ collisions, whereas most of the $\mathrm{e}^{+} \mathrm{e}^{-}$pairs originate from open-beauty hadron decays for $p_{\mathrm{T}, \text { ee }}>3.5 \mathrm{GeV} / c$. The contribution from $\mathrm{J} / \psi$ decays is small over the whole $p_{\mathrm{T}, \text { ee }}$ range. The dielectron production in $\mathrm{pp}$ and $\mathrm{p}-\mathrm{Pb}$ collisions at $\sqrt{s_{\mathrm{NN}}}=5.02 \mathrm{TeV}$ is well described by the hadronic cocktail, utilizing heavy-flavor cross sections fitted to the pp data and assuming a scaling of the heavy-flavor cross sections with the $A$ of the $\mathrm{Pb}$ nucleus. In particular, no significant modification of the heavy-flavor production in the measured kinematic regions is justified by the analysis of the $\mathrm{p}-\mathrm{Pb}$ collisions data.

\section{Nuclear modification factor}

The nuclear modification factor, $R_{\mathrm{pPb}}$, is calculated as

$$
R_{\mathrm{pPb}}\left(m_{\mathrm{ee}}\right)=\frac{1}{A} \frac{d \sigma_{e e}^{\mathrm{pPb}} / d m_{\mathrm{ee}}}{d \sigma_{\mathrm{ee}}^{\mathrm{pp}} / d m_{\mathrm{ee}}},
$$

with $\sigma_{\mathrm{ee}}^{\mathrm{pPb}}$ and $\sigma_{\mathrm{ee}}^{\mathrm{pp}}$ representing the cross sections of dielectron production in $\mathrm{p}-\mathrm{Pb}$ and $\mathrm{pp}$ collisions, respectively, and $A$ denoting the mass number of the $\mathrm{Pb}$ nucleus (208). The $R_{\mathrm{pPb}}$ allows for a direct comparison of the measurements in the $\mathrm{pp}$ and $\mathrm{p}-\mathrm{Pb}$ collision systems. The systematic uncertainties of the $\mathrm{p}-\mathrm{Pb}$ and $\mathrm{pp}$ measurements are treated as independent and, thus, added in quadrature. The dielectron $R_{\mathrm{pPb}}$ as a function of $m_{\mathrm{ee}}$ for $p_{\mathrm{T}, \text { ee }}<8 \mathrm{GeV} / c$ is shown in Fig. 8 . The data are compared to the $R_{\mathrm{pPb}}$ of the hadronic cocktails as described in Sec. IV (solid black line). In the cocktail $R_{\mathrm{pPb}}$, the uncertainties from the open heavy-flavor contributions as well as those from the scaling factor applied to the $\pi^{ \pm}$ parametrizations, the $\rho / \pi^{ \pm}, \omega / \pi^{ \pm}$, and $\eta / \pi^{0} p_{\mathrm{T}}$ ratios are 

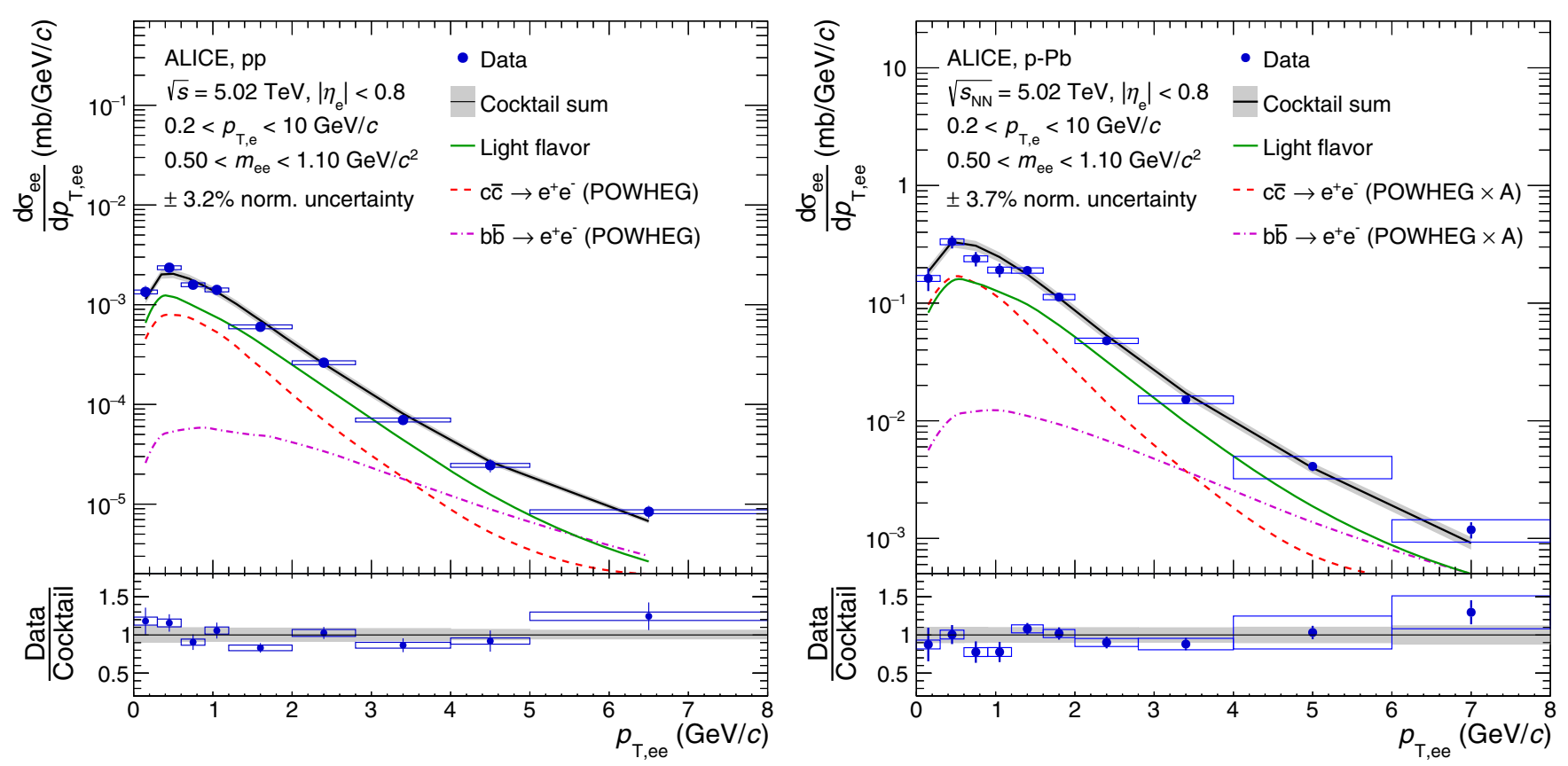

FIG. 6. Differential $\mathrm{e}^{+} \mathrm{e}^{-}$cross section as a function of $p_{\mathrm{T}, \mathrm{ee}}$ in the low-mass region measured in $\mathrm{pp}$ (left) and $\mathrm{p}-\mathrm{Pb}$ (right) collisions at $\sqrt{s_{\mathrm{NN}}}=5.02 \mathrm{TeV}$. The data are compared to the hadronic cocktail, where the heavy-flavor contributions are fitted to the pp spectrum in the intermediate-mass region, and for $\mathrm{p}-\mathrm{Pb}$ collisions scaled with the atomic mass number of the $\mathrm{Pb}$ nucleus $A=208$. The gray band represents the total uncertainty on the hadronic cocktail.

fully correlated, and therefore cancel out. The uncertainties on the parametrized $\pi, \phi$, and $\mathrm{J} / \psi$ spectra are propagated to the $R_{\mathrm{pPb}}$. Since they are based on independent measurements they are added quadratically. The measured $R_{\mathrm{pPb}}$ is below the expectation of binary collision scaling for $m_{\mathrm{ee}}<1.1 \mathrm{GeV} / c^{2}$, where the fraction of dielectrons from light-flavor hadron decays to the total expected $\mathrm{e}^{+} \mathrm{e}^{-}$cross section in $\mathrm{p}-\mathrm{Pb}$ collisions, denoted by the green area, is not negligible. The
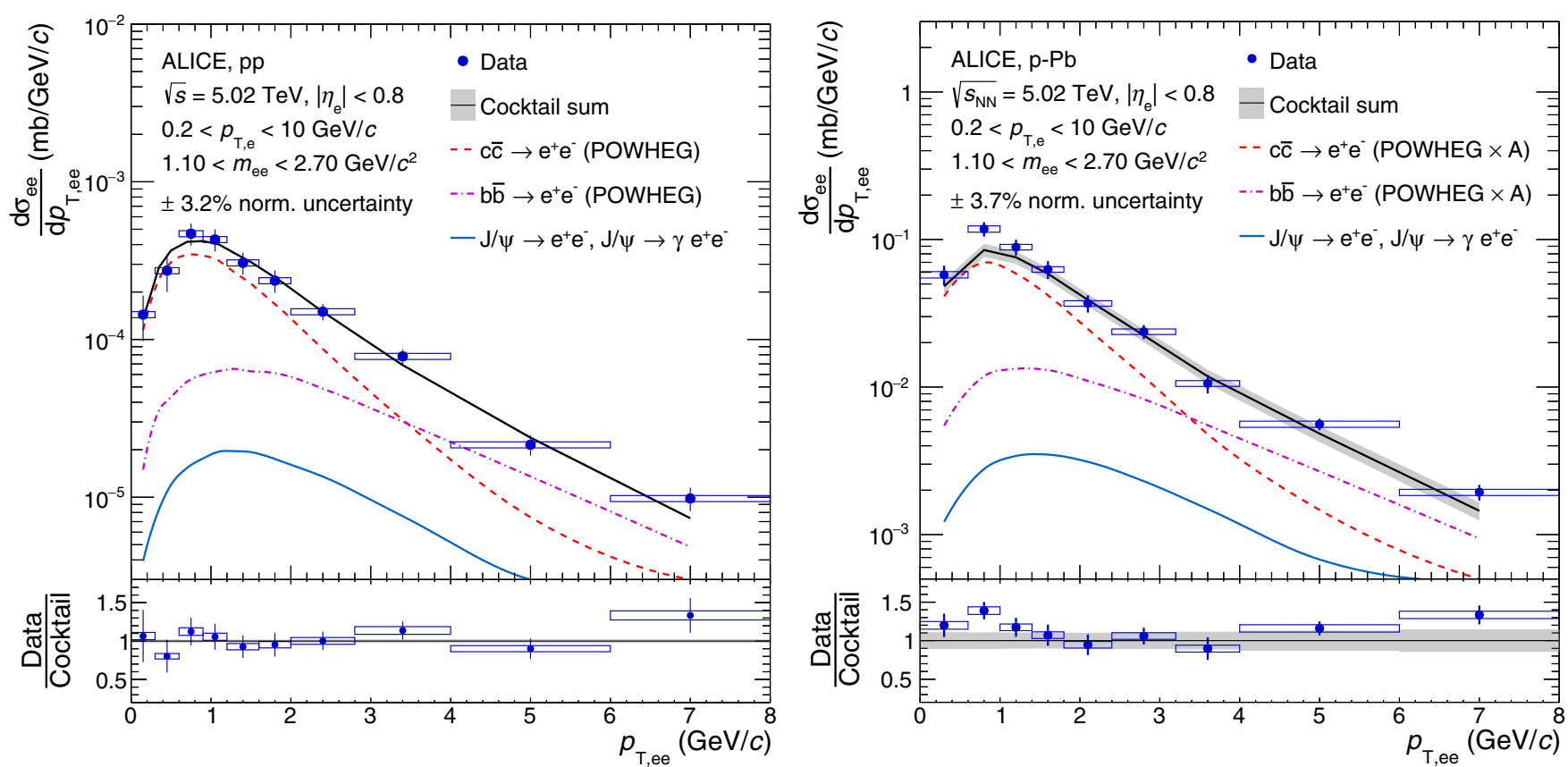

FIG. 7. Differential $\mathrm{e}^{+} \mathrm{e}^{-}$cross section as a function of $p_{\mathrm{T}, \mathrm{ee}}$ in the intermediate-mass region measured in $\mathrm{pp}$ (left) and $\mathrm{p}-\mathrm{Pb}$ (right) collisions at $\sqrt{s_{\mathrm{NN}}}=5.02 \mathrm{TeV}$. The data are compared to the hadronic cocktail, where the heavy-flavor contributions are fitted to the $\mathrm{pp}$ spectrum in the intermediate-mass region, and for $\mathrm{p}-\mathrm{Pb}$ collisions scaled with the atomic mass number of the $\mathrm{Pb}$ nucleus $A=208$. The gray band represents the total uncertainty on the hadronic cocktail. 


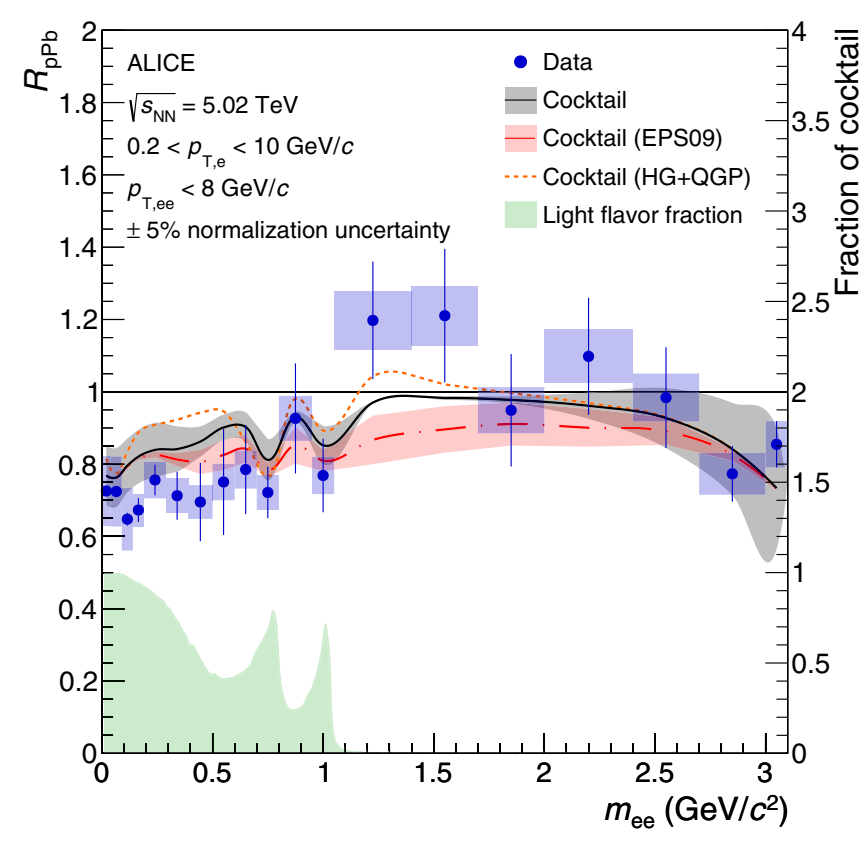

FIG. 8. Measured dielectron nuclear modification factor as a function of $m_{\mathrm{ee}}$ at $\sqrt{s_{\mathrm{NN}}}=5.02 \mathrm{TeV}$. The data are shown in blue, with their statistical and systematic uncertainties depicted as vertical bars and boxes. The baseline expectation, calculated from the pp and $\mathrm{p}-\mathrm{Pb}$ cocktails outlined in Sec. IV, is shown as a black line with a gray band indicating its uncertainties. Two additional cocktails, one incorporating a modified charm production due to CNM effects and another one including thermal radiation from the hadronic and partonic phases, are shown as red and orange dashed lines, respectively.

$R_{\mathrm{pPb}}$ is consistent with unity in the IMR within uncertainties, displaying a step between the two mass regions. The behavior is reproduced, within uncertainties, by the hadronic cocktail assuming no further modification of the open heavy-flavor cross sections beyond binary collision scaling. This suggests a different scaling behavior of the light-flavor production from binary collision scaling, as already indicated in previous measurements [97].

An additional cocktail calculation incorporating a modification of the open-charm contribution via CNM effects is shown by a dashed red line in Fig. 8. The CNM effects on the production of dielectrons from open-charm hadron decays are incorporated by using the EPS09 nPDF [18] in the POWHEG calculations. In the mass region below $1 \mathrm{GeV} / c^{2}$, where the admixture of charm is significant, the modification of the charm contribution improves the description of the measured $R_{\mathrm{pPb}}$. In the IMR, the data are just beyond the upper edge of the systematic uncertainties of the calculations including CNM for the charm production. On the one hand, it suggests negligible CNM effects compared to the current precision of the measurement in this mass range, where the $p_{\mathrm{T}}$ of $\mathrm{D}$ mesons, from which the dielectrons originate, is larger than $2 \mathrm{GeV} / c$ according to calculations performed with PYTHIA 6. This is in agreement with previous results on the $\mathrm{D}$ meson $R_{\mathrm{pPb}}$ at $\sqrt{s_{\mathrm{NN}}}=5.02 \mathrm{TeV}$ by ALICE, which show no significant modification of the $p_{\mathrm{T}}$ spectra above $2 \mathrm{GeV} / c[27,28]$ compared to $\mathrm{pp}$ collisions. The dielectron cross section from charm at lower $m_{\mathrm{ee}}$ however is sensitive to the production of low $p_{\mathrm{T}} \mathrm{D}$ mesons $\left(p_{\mathrm{T}}<2 \mathrm{GeV} / c\right)$. On the other hand, a possible additional source of electron pairs in $\mathrm{p}-\mathrm{Pb}$ collisions compared to pp collisions could compensate CNM effects on the heavy-flavor production.

The measured $R_{\mathrm{pPb}}$ is further compared to calculations including thermal radiation from the hadronic and partonic phases, based on a model which describes the dilepton enhancement measured in heavy-ion collisions at the SPS and RHIC $[7,51,52,98]$. The contribution of thermal dielectrons is obtained from an expanding thermal fireball model for $\mathrm{p}-\mathrm{Pb}$ collisions at $\sqrt{s_{\mathrm{NN}}}=5.02 \mathrm{TeV}$, corresponding to a mean charged-particle multiplicity at midrapidity of $\left\langle d N_{\mathrm{ch}} / d y\right\rangle=20$, corrected for weak decay feeddown. The equation of state was extracted from lattice QCD computations with a crossover transition around the critical temperature $T_{\mathrm{c}}=170 \mathrm{MeV}$. A broadening of the $\rho$ electromagnetic spectral function is expected as an effect of interactions in the hot hadronic phase. The thermal emission rate of dielectrons from the hadronic phase is calculated based on the hadronic many-body theory. The effects of the detector resolution are not included in the calculations and no modification of the heavy-flavor contribution is considered. A hadronic cocktail including these calculations is shown as the orange dotted line (HG+QGP). In the range $0.2<m_{\mathrm{ee}}<0.6 \mathrm{GeV} / c^{2}$, the model tends to slightly overestimate the measured $R_{\mathrm{pPb}}$, whereas in the IMR it agrees with the data within their uncertainties. An additional thermal source of dielectrons in $\mathrm{p}-\mathrm{Pb}$ collisions compared to pp collisions can not be excluded by the data.

To further investigate the modifications of the open-charm contribution to the $\mathrm{e}^{+} \mathrm{e}^{-}$spectrum, the dielectron $R_{\mathrm{pPb}}$ as a function of $p_{\mathrm{T}, \mathrm{ee}}$ is shown in the LMR and IMR in Fig. 9.

In the LMR, the fraction of $\mathrm{e}^{+} \mathrm{e}^{-}$pairs from light-flavor hadron decays ranges from about $40 \%$ to $60 \%$ depending on $p_{\mathrm{T}, \text { ee }}$. For $p_{\mathrm{T} \text {,ee }}$ larger than about $1 \mathrm{GeV} / c$ the data are compatible with binary collision scaling, indicating that the production of light-flavor hadrons is driven by the initial hard scatterings of the incoming partons and is not affected by CNM effects. This no longer holds true for $p_{\mathrm{T}, \mathrm{ee}}<1 \mathrm{GeV} / c$, pointing to a change in the production mechanism of the light-flavor hadrons. These features can be reproduced by the hadronic cocktail. Inclusion of CNM effects for the charm contribution in the hadronic cocktail only have a small effect. The uncertainties on the data as well as the CNM calculations themselves are too large to draw any conclusion. The addition of the thermal contributions in the LMR is disfavored by the data at low- $p_{\mathrm{T}, \text { ee }}\left(p_{\mathrm{T}, \text { ee }}<1 \mathrm{GeV} / c\right)$, whereas at higher $p_{\mathrm{T} \text {,ee }}$ the uncertainties on the data do not allow for any discrimination between the three models.

In the IMR, the contribution from light-flavor hadron decays is negligible. The $R_{\mathrm{pPb}}$ is consistent with unity, indicating that the heavy-flavor cross sections approximately scale with the number of binary collisions in this range. According to the calculations using EPS09 nPDFs, a suppression of the total $\mathrm{e}^{+} \mathrm{e}^{-}$cross section is expected for $p_{\mathrm{T}, \mathrm{ee}}<3.5 \mathrm{GeV} / c$ due to CNM effects on dielectrons from open-charm hadron decays. Nevertheless, it is disfavored by these data. On the contrary, the cocktail calculation including thermal contributions would be preferred by the data. In particular, for $p_{\mathrm{T} \text {, ee }}<1 \mathrm{GeV} / c$ 

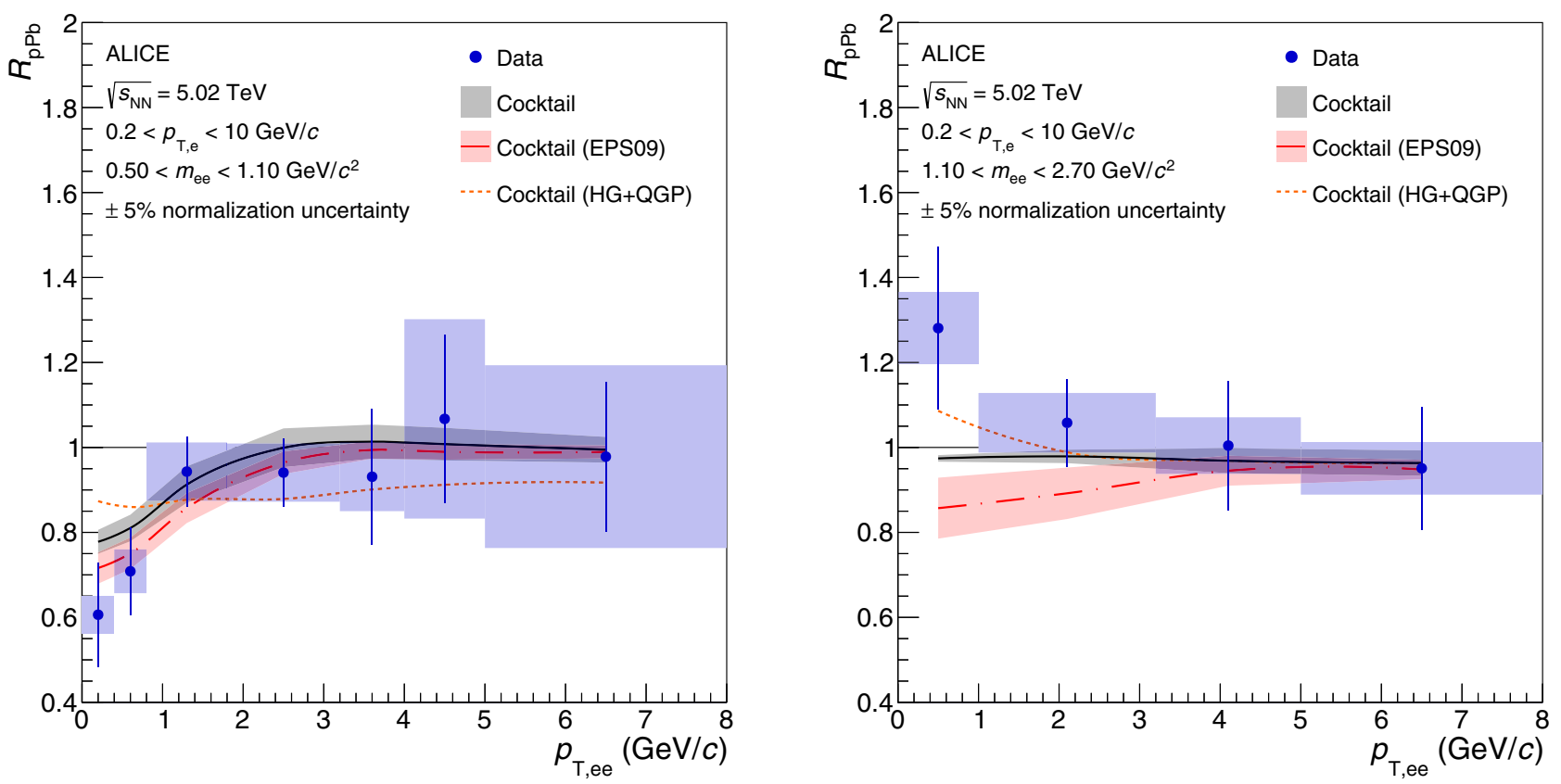

FIG. 9. Measured dielectron nuclear modification factor as a function of $p_{\mathrm{T} \text {,ee }}$ in the low-mass region (left) and intermediate-mass region (right) at $\sqrt{s_{\mathrm{NN}}}=5.02 \mathrm{TeV}$. The data are shown in blue, with their statistical and systematic uncertainties depicted as vertical bars and boxes. The baseline expectation, calculated from the $\mathrm{pp}$ and $\mathrm{p}-\mathrm{Pb}$ cocktails outlined in Sec. IV, is shown as a black line with a gray band indicating its uncertainties. Two additional cocktails, one incorporating a modified charm production due to CNM effects and another one including thermal radiation from the hadronic and partonic phases, are shown as red and orange dashed lines, respectively.

a thermal contribution significantly helps to improve the description of the data.

Finally, a potential interplay between CNM effects and the thermal contribution cannot be ruled out. Therefore, it is mandatory to separate the dielectrons from heavy-flavor hadron decays and those from thermal radiation. This could be achieved by an analysis as a function of the distance-of-closest approach of the $\mathrm{e}^{+} \mathrm{e}^{-}$pairs to the collision vertex [14].

\section{CONCLUSIONS}

The dielectron production at midrapidity $\left(\left|\eta_{\mathrm{e}}\right|<0.8\right)$ was measured with the ALICE detector as a function of invariant mass and pair transverse momentum in $\mathrm{pp}$ and $\mathrm{p}-\mathrm{Pb}$ collisions at $\sqrt{s_{\mathrm{NN}}}=5.02 \mathrm{TeV}$.

In pp collisions, the dielectron continuum can be well described by the expected contributions from light-flavor hadron decays and calculations of $\mathrm{e}^{+} \mathrm{e}^{-}$pairs from heavyflavor hadron decays fitted to the data. The cross sections of $c \bar{c}$ and $b \bar{b}$ production at midrapidity are extracted from the measurement by a double-differential fit to the $m_{\mathrm{ee}}$ and $p_{\mathrm{T}, \text { ee }}$ spectrum in the intermediate-mass region. Templates from two different event generators, PYTHIA 6 [66] and POWHEG [87-90], are used. Both calculations can describe the data well, yet they yield significantly different results for the cross sections of the single $c \bar{c}$ and $b \bar{b}$ contributions. The hadronization of c- and b-quarks as well as the decay of the heavy-flavor hadrons is done in both PYTHIA 6 and POWHEG simulations with the Perugia 2011 tune of PYTHIA $6.4[66,86]$. Therefore, the model dependence of the extracted cross sections directly reflects the sensitivity of the dielectron measurement to the different implementation of the heavy-quark production mechanisms in the Monte Carlo event generators. The measured $d \sigma_{\mathrm{c} \overline{\mathrm{c}}} /\left.d y\right|_{y=0}$ and $d \sigma_{\mathrm{b} \overline{\mathrm{b}}} /\left.d y\right|_{y=0}$ are compared to existing results from dielectron measurements, as well as measurements of identified charm hadrons and semileptonic decays of beauty hadrons, in pp collisions at different $\sqrt{s}$. The difference between the cross sections extracted in this analysis with the two event generators is comparable to those reported in previous observations at $\sqrt{s}=7$ and $13 \mathrm{TeV}$. The slope of the center-of-mass energy dependence of the cross sections can be described by FONLL calculations.

The dielectron $m_{\mathrm{ee}}$ and $p_{\mathrm{T} \text {,ee }}$ spectra in $\mathrm{p}-\mathrm{Pb}$ collisions at $\sqrt{s_{\mathrm{NN}}}=5.02 \mathrm{TeV}$, reported here for the first time, are compared to a hadronic cocktail composed of the expected dielectron cross sections from the known hadron decays. Whereas $\mathrm{e}^{+} \mathrm{e}^{-}$pairs from light-flavor and $\mathrm{J} / \psi$ hadron decays are estimated using independent measurements of hadrons, the contributions of dielectrons from open heavy-flavor hadron decays are determined from the dielectron measurement in pp collisions at the same center-of-mass energy using POWHEG as the event generator. The heavy-flavor cross sections are assumed to scale with the atomic mass number of the $\mathrm{Pb}$ nucleus in $\mathrm{p}-\mathrm{Pb}$ collisions, with respect to the measured $\mathrm{pp}$ reference. Good agreement is observed between the measured and expected total $\mathrm{e}^{+} \mathrm{e}^{-}$cross section.

The dielectron $R_{\mathrm{pPb}}$ as a function of $m_{\mathrm{ee}}$ highlights the different scaling behavior of the light- and heavy-flavor dielectron sources. While the measured $R_{\mathrm{pPb}}$ is below one for $m_{\mathrm{ee}}<1 \mathrm{GeV} / c^{2}$, it is consistent with unity within uncertainties in the IMR where most of the $\mathrm{e}^{+} \mathrm{e}^{-}$pairs originate from correlated open heavy-flavor hadron decays. 
On the one hand, calculations including a suppression of the charm production using the nPDF EPS09 do not describe the data as well as the hadronic cocktail using the atomic mass number scaling hypothesis in the intermediate-mass region. The central value of the computations including CNM effects is nevertheless closer to the measured $R_{\mathrm{pPb}}$ at masses around $0.5 \mathrm{GeV} / c^{2}$. On the other hand, including a thermal contribution from a hot hadronic and partonic phase to the dielectron cocktail helps in the description of the data in the IMR. The thermal radiation calculations seem however to overestimate the production of dielectrons in the LMR. The hadronic cocktail calculations including CNM effects and thermal radiation show that both play a role at low $p_{\mathrm{T} \text {,ee }}$ with opposite trends, although the current uncertainties on the measured $p_{\mathrm{T} \text {,ee }}$ dependence of $R_{\mathrm{pPb}}$ are still too large to reject any of the calculations presented. Moreover, CNM effects on the charm production and thermal radiation from a hot medium possibly formed in $\mathrm{p}-\mathrm{Pb}$ collisions could cancel each other, if both are present, which makes it necessary to disentangle them in a more sophisticated approach.

A more detailed study of the dielectron production in $\mathrm{p}-\mathrm{Pb}$ collisions requires the separation of $\mathrm{e}^{+} \mathrm{e}^{-}$pairs from prompt sources and those from the displaced open heavyflavor hadron decays. The distance-of-closest approach of the $\mathrm{e}^{+} \mathrm{e}^{-}$pair to the collision vertex, pioneered at the LHC by ALICE in the dielectron analysis of the $\mathrm{pp}$ data at $\sqrt{s}=7 \mathrm{TeV}$ [14], could enable the search for the presence of a possible additional contribution from thermal radiation in $\mathrm{p}-\mathrm{Pb}$ collisions, in particular in high-multiplicity events. In the near future, the dielectron analysis will greatly benefit from the upgrades of the ALICE TPC [99,100], the ITS [101] and a completely new readout system [102] and computing framework [103]. The data acquisition rate will increase by a factor of 100, while the pointing resolution of primary tracks will improve by a factor of 3 to 6 , depending on their orientation with respect to the magnetic field. This will open up the possibility to study the dielectron production with unprecedented precision and detail.

\section{ACKNOWLEDGMENTS}

The ALICE Collaboration thanks all its engineers and technicians for their invaluable contributions to the construction of the experiment and the CERN accelerator teams for the outstanding performance of the LHC complex. The ALICE Collaboration gratefully acknowledges the resources and support provided by all Grid centres and the Worldwide LHC Computing Grid (WLCG) collaboration. The ALICE Collaboration acknowledges the following funding agencies for their support in building and running the ALICE detector: A. I. Alikhanyan National Science Laboratory (Yerevan Physics Institute) Foundation (ANSL), State Committee of Science and World Federation of Scientists (WFS), Armenia; Austrian Academy of Sciences, Austrian Science Fund (FWF): [M 2467-N36] and Nationalstiftung für Forschung, Technologie und Entwicklung, Austria; Ministry of Communications and High Technologies, National Nuclear Research Center, Azerbaijan; Conselho Nacional de Desenvolvimento
Científico e Tecnológico (CNPq), Financiadora de Estudos e Projetos (Finep), Fundação de Amparo à Pesquisa do Estado de São Paulo (FAPESP) and Universidade Federal do Rio Grande do Sul (UFRGS), Brazil; Ministry of Education of China (MOEC), Ministry of Science \& Technology of China (MSTC) and National Natural Science Foundation of China (NSFC), China; Ministry of Science and Education and Croatian Science Foundation, Croatia; Centro de Aplicaciones Tecnológicas y Desarrollo Nuclear (CEADEN), Cubaenergía, Cuba; Ministry of Education, Youth and Sports of the Czech Republic, Czech Republic; The Danish Council for Independent Research | Natural Sciences, the VILLUM FONDEN and Danish National Research Foundation (DNRF), Denmark; Helsinki Institute of Physics (HIP), Finland; Commissariat à l'Energie Atomique (CEA) and Institut National de Physique Nucléaire et de Physique des Particules (IN2P3) and Centre National de la Recherche Scientifique (CNRS), France; Bundesministerium für Bildung und Forschung (BMBF) and GSI Helmholtzzentrum für Schwerionenforschung $\mathrm{GmbH}$, Germany; General Secretariat for Research and Technology, Ministry of Education, Research and Religions, Greece; National Research, Development and Innovation Office, Hungary; Department of Atomic Energy Government of India (DAE), Department of Science and Technology, Government of India (DST), University Grants Commission, Government of India (UGC) and Council of Scientific and Industrial Research (CSIR), India; Indonesian Institute of Science, Indonesia; Centro Fermi - Museo Storico della Fisica e Centro Studi e Ricerche Enrico Fermi and Istituto Nazionale di Fisica Nucleare (INFN), Italy; Institute for Innovative Science and Technology, Nagasaki Institute of Applied Science (IIST), Japanese Ministry of Education, Culture, Sports, Science and Technology (MEXT) and Japan Society for the Promotion of Science (JSPS) KAKENHI, Japan; Consejo Nacional de Ciencia (CONACYT) y Tecnología, through Fondo de Cooperación Internacional en Ciencia y Tecnología (FONCICYT) and Dirección General de Asuntos del Personal Academico (DGAPA), Mexico; Nederlandse Organisatie voor Wetenschappelijk Onderzoek (NWO), Netherlands; The Research Council of Norway, Norway; Commission on Science and Technology for Sustainable Development in the South (COMSATS), Pakistan; Pontificia Universidad Católica del Perú, Peru; Ministry of Science and Higher Education, National Science Centre and WUT ID-UB, Poland; Korea Institute of Science and Technology Information and National Research Foundation of Korea (NRF), Republic of Korea; Ministry of Education and Scientific Research, Institute of Atomic Physics and Ministry of Research and Innovation and Institute of Atomic Physics, Romania; Joint Institute for Nuclear Research (JINR), Ministry of Education and Science of the Russian Federation, National Research Centre Kurchatov Institute, Russian Science Foundation and Russian Foundation for Basic Research, Russia; Ministry of Education, Science, Research and Sport of the Slovak Republic, Slovakia; National Research Foundation of South Africa, South Africa; Swedish Research Council (VR) and Knut \& Alice Wallenberg Foundation (KAW), Sweden; European Organization for Nuclear Research, Switzerland; Suranaree University of Technology (SUT), National Science and Technology 
Development Agency (NSDTA) and Office of the Higher Education Commission under NRU project of Thailand, Thailand; Turkish Atomic Energy Agency (TAEK), Turkey; National Academy of Sciences of Ukraine, Ukraine; Science and Tech- nology Facilities Council (STFC), United Kingdom; National Science Foundation of the USA (NSF) and United States Department of Energy, Office of Nuclear Physics (DOE NP), USA.
[1] K. Aamodt et al. (ALICE Collaboration), The ALICE experiment at the CERN LHC, JINST 3, S08002 (2008).

[2] F. Karsch and M. Lutgemeier, Deconfinement and chiral symmetry restoration in an $\mathrm{SU}(3)$ gauge theory with adjoint fermions, Nucl. Phys. B 550, 449 (1999).

[3] R. Rapp, J. Wambach, and H. van Hees, The chiral restoration transition of QCD and low mass dileptons, in Relativistic Heavy Ion Physics, edited by R. Stock, Landolt-Börnstein - Group I Elementary Particles, Nuclei and Atoms Vol. 23 (Springer-Verlag, Berlin-Heidelberg, 2010).

[4] I. Tserruya, Electromagnetic probes, in Relativistic Heavy Ion Physics edited by R. Stock, Landolt-Börnstein - Group I Elementary Particles, Nuclei and Atoms Vol. 23 (Springer-Verlag, Berlin-Heidelberg, 2010).

[5] G. Agakichiev et al. (CERES Collaboration), Enhanced Production of Low Mass Electron Pairs in 200-GeV/u S-Au Collisions at the CERN SPS, Phys. Rev. Lett. 75, 1272 (1995).

[6] D. Adamova et al. (CERES Collaboration), Modification of the $\rho$-meson detected by low-mass electron-positron pairs in central $\mathrm{Pb}-\mathrm{Au}$ collisions at $158-\mathrm{A}-\mathrm{GeV} / \mathrm{c}$, Phys. Lett. B 666, 425 (2008).

[7] R. Arnaldi et al. (NA60 Collaboration), First Measurement of the $\rho$ Spectral Function in High-Energy Nuclear Collisions, Phys. Rev. Lett. 96, 162302 (2006).

[8] R. Arnaldi et al. (NA60 Collaboration), Evidence for Radial Flow of Thermal Dileptons in High-Energy Nuclear Collisions, Phys. Rev. Lett. 100, 022302 (2008).

[9] A. Adare et al. (PHENIX Collaboration), Dielectron production in $\mathrm{Au}+\mathrm{Au}$ collisions at $\sqrt{s_{\mathrm{NN}}}=200 \mathrm{GeV}$, Phys. Rev. C 93, 014904 (2016).

[10] L. Adamczyk et al. (STAR Collaboration), Dielectron Mass Spectra from $\mathrm{Au}+\mathrm{Au}$ Collisions at $\sqrt{s_{\mathrm{NN}}}=200 \mathrm{GeV}$ Phys. Rev. Lett. 113, 022301 (2014).

[11] S. Acharya et al. (ALICE Collaboration), Measurement of dielectron production in central $\mathrm{Pb}-\mathrm{Pb}$ collisions at $\sqrt{s_{\mathrm{NN}}}=$ 2.76 TeV, Phys. Rev. C 99, 024002 (2019).

[12] A. Adare et al. (PHENIX Collaboration), Cross section for $b \bar{b}$ production via dielectrons in $d+$ Au collisions at $\sqrt{s_{\mathrm{NN}}}=$ $200 \mathrm{GeV}$, Phys. Rev. C 91, 014907 (2015).

[13] A. Adare et al. (PHENIX Collaboration), Measurements of $e^{+} e^{-}$pairs from open heavy flavor in $p+p$ and $d+A$ collisions at $\sqrt{s_{\mathrm{NN}}}=200 \mathrm{GeV}$, Phys. Rev. C 96, 024907 (2017).

[14] S. Acharya et al. (ALICE Collaboration), Dielectron production in proton-proton collisions at $\sqrt{s}=7 \mathrm{TeV}$, J. High Energy Phys. 09 (2018) 064.

[15] S. Acharya et al. (ALICE Collaboration), Dielectron and heavy-quark production in inelastic and high-multiplicity proton-proton collisions at $\sqrt{s}=13 \mathrm{TeV}$, Phys. Lett. B 788, 505 (2019).

[16] M. Cacciari, M. Greco, and P. Nason, The $p_{T}$ spectrum in heavy flavor hadroproduction, J. High Energy Phys. 05 (1998) 007.

[17] M. Hirai, S. Kumano, and T. H. Nagai, Determination of nuclear parton distribution functions and their uncertainties in next-to-leading order, Phys. Rev. C 76, 065207 (2007).
[18] K. J. Eskola, H. Paukkunen, and C. A. Salgado, EPS09: A new generation of NLO and LO nuclear parton distribution functions, J. High Energy Phys. 04 (2009) 065.

[19] K. J. Eskola, P. Paakkinen, H. Paukkunen, and C. A. Salgado, EPPS16: Nuclear parton distributions with LHC data, Eur. Phys. J. C 77, 163 (2017).

[20] F. Gelis, E. Iancu, J. Jalilian-Marian, and R. Venugopalan, The color glass condensate, Annu. Rev. Nucl. Part. Sci. 60, 463 (2010).

[21] P. Tribedy and R. Venugopalan, QCD saturation at the LHC: Comparisons of models to $\mathrm{p}+\mathrm{p}$ and $\mathrm{A}+\mathrm{A}$ data and predictions for $\mathrm{p}+\mathrm{Pb}$ collisions, Phys. Lett. B 710, 125 (2012); 718, 1154 (2013).

[22] J. L. Albacete, A. Dumitru, H. Fujii, and Y. Nara, CGC predictions for $\mathrm{p}+\mathrm{Pb}$ collisions at the LHC, Nucl. Phys. A 897, 1 (2013).

[23] A. H. Rezaeian, CGC predictions for $\mathrm{p}+\mathrm{A}$ collisions at the LHC and signature of QCD saturation, Phys. Lett. B 718, 1058 (2013).

[24] N. Armesto, Nuclear shadowing, J. Phys. G 32, R367 (2006).

[25] I. Vitev, Non-Abelian energy loss in cold nuclear matter, Phys. Rev. C 75, 064906 (2007).

[26] B. Z. Kopeliovich, J. Nemchik, A. Schafer, and A. V. Tarasov, Cronin Effect in Hadron Production Off Nuclei, Phys. Rev. Lett. 88, 232303 (2002).

[27] J. Adam et al. (ALICE Collaboration), D-meson production in $p$ - $\mathrm{Pb}$ collisions at $\sqrt{s_{\mathrm{NN}}}=5.02 \mathrm{TeV}$ and in pp collisions at $\sqrt{s}=7$ TeV, Phys. Rev. C 94, 054908 (2016).

[28] S. Acharya et al. (ALICE Collaboration), Measurement of prompt $\mathrm{D}^{0}, \mathrm{D}^{+}, \mathrm{D}^{*+}$, and $\mathrm{D}_{\mathrm{S}}^{+}$production in $\mathrm{p}-\mathrm{Pb}$ collisions at $\sqrt{\mathrm{s}_{\mathrm{NN}}}=5.02 \mathrm{TeV}$, J. High Energy Phys. 12 (2019) 092.

[29] J. Adam et al. (ALICE Collaboration), Measurement of electrons from heavy-flavor hadron decays in $\mathrm{p}-\mathrm{Pb}$ collisions at $\sqrt{s_{\mathrm{NN}}}=5.02 \mathrm{TeV}$, Phys. Lett. B 754, 81 (2016).

[30] S. Acharya et al. (ALICE Collaboration), Measurement of electrons from semileptonic heavy-flavour hadron decays at midrapidity in $\mathrm{pp}$ and $\mathrm{Pb}-\mathrm{Pb}$ collisions at $\sqrt{s_{\mathrm{NN}}}=5.02 \mathrm{TeV}$, Phys. Lett. B 804, 135377 (2020).

[31] J. Adam et al. (ALICE Collaboration), Measurement of azimuthal correlations of $\mathrm{D}$ mesons and charged particles in pp collisions at $\sqrt{s}=7 \mathrm{TeV}$ and $\mathrm{p}-\mathrm{Pb}$ collisions at $\sqrt{s_{\mathrm{NN}}}=$ 5.02 TeV, Eur. Phys. J. C 77, 245 (2017).

[32] S. Acharya et al. (ALICE Collaboration), Prompt and non-prompt $\mathrm{J} / \psi$ production and nuclear modification at midrapidity in $\mathrm{p}-\mathrm{Pb}$ collisions at $\sqrt{\mathbf{s}_{\mathrm{NN}}}=5.02 \mathrm{TeV}$, Eur. Phys. J. C 78, 466 (2018).

[33] R. Aaij et al. (LHCb Collaboration), Study of prompt $\mathrm{D}^{0}$ meson production in $\mathrm{p}-\mathrm{Pb}$ collisions at $\sqrt{s_{\mathrm{NN}}}=5 \mathrm{TeV}$, J. High Energy Phys. 10 (2017) 090.

[34] R. Aaij et al. (LHCb Collaboration), Prompt and nonprompt $\mathrm{J} / \psi$ production and nuclear modification in $\mathrm{pPb}$ collisions at $\sqrt{s_{\mathrm{NN}}}=8.16 \mathrm{TeV}$, Phys. Lett. B 774, 159 (2017).

[35] S. Acharya et al. (ALICE Collaboration), Production of muons from heavy-flavour hadron decays in $\mathrm{p}-\mathrm{Pb}$ collisions at $\sqrt{s_{\mathrm{NN}}}=5.02 \mathrm{TeV}$, Phys. Lett. B 770, 459 (2017). 
[36] S. Chatrchyan et al. (CMS Collaboration), Observation of long-range near-side angular correlations in proton-lead collisions at the LHC, Phys. Lett. B 718, 795 (2013).

[37] B. Abelev et al. (ALICE Collaboration), Long-range angular correlations on the near and away side in $p-\mathrm{Pb}$ collisions at $\sqrt{s_{\mathrm{NN}}}=5.02 \mathrm{TeV}$, Phys. Lett. B 719, 29 (2013).

[38] B. Abelev et al. (ALICE Collaboration), Long-range angular correlations of $\pi, \mathrm{K}$ and $\mathrm{p}$ in $\mathrm{p}-\mathrm{Pb}$ collisions at $\sqrt{s_{\mathrm{NN}}}=$ $5.02 \mathrm{TeV}$, Phys. Lett. B 726, 164 (2013).

[39] G. Aad et al. (ATLAS Collaboration), Observation of Associated Near-Side and Away-Side Long-Range Correlations in $\sqrt{s_{\mathrm{NN}}}=5.02 \mathrm{TeV}$ Proton-Lead Collisions with the ATLAS Detector, Phys. Rev. Lett. 110, 182302 (2013).

[40] J. Adam et al. (ALICE Collaboration), Forward-central twoparticle correlations in $\mathrm{p}-\mathrm{Pb}$ collisions at $\sqrt{s_{\mathrm{NN}}}=5.02 \mathrm{TeV}$, Phys. Lett. B 753, 126 (2016).

[41] A. M. Sirunyan et al. (CMS Collaboration), Elliptic Flow of Charm and Strange Hadrons in High-Multiplicity $\mathrm{pPb}$ Collisions at $\sqrt{s_{\mathrm{NN}}}=8.16 \mathrm{TeV}$, Phys. Rev. Lett. 121, 082301 (2018).

[42] S. Acharya et al. (ALICE Collaboration), Azimuthal Anisotropy of Heavy-Flavor Decay Electrons in $p$-Pb Collisions at $\sqrt{s_{\mathrm{NN}}}=5.02 \mathrm{TeV}$, Phys. Rev. Lett. 122, 072301 (2019).

[43] V. Khachatryan et al. (CMS Collaboration), Evidence for Collective Multiparticle Correlations in $\mathrm{p}-\mathrm{Pb}$ Collisions, Phys. Rev. Lett. 115, 012301 (2015).

[44] M. Aaboud et al. (ATLAS Collaboration), Measurement of long-range multiparticle azimuthal correlations with the subevent cumulant method in $p p$ and $p+P b$ collisions with the ATLAS detector at the CERN Large Hadron Collider, Phys. Rev. C 97, 024904 (2018).

[45] B. Abelev et al. (ALICE Collaboration), Multiplicity dependence of $\pi, \mathrm{K}, \mathrm{p}$, and $\Lambda$ production in $\mathrm{p}-\mathrm{Pb}$ collisions at $\sqrt{s_{\mathrm{NN}}}=5.02 \mathrm{TeV}$, Phys. Lett. B 728, 25 (2014).

[46] S. Chatrchyan et al. (CMS Collaboration), Study of the production of $\pi^{ \pm}, \mathrm{K}$, and $\mathrm{p}$ in $\mathrm{pPb}$ collisions at $\sqrt{s_{\mathrm{NN}}}=5.02 \mathrm{TeV}$, Eur. Phys. J. C 74, 2847 (2014).

[47] J. Adam et al. (ALICE Collaboration), Enhanced production of multi-strange hadrons in high-multiplicity proton-proton collisions, Nat. Phys. 13, 535 (2017).

[48] B. Abelev et al. (ALICE Collaboration), Suppression of $\psi(2 \mathrm{~S})$ production in $\mathrm{p}-\mathrm{Pb}$ collisions at $\sqrt{s_{\mathrm{NN}}}=5.02 \mathrm{TeV}$, J. High Energy Phys. 12 (2014) 073.

[49] R. Aaij et al. (LHCb Collaboration), Study of $\psi(2 S)$ production and cold nuclear matter effects in $\mathrm{pPb}$ collisions at $\sqrt{s_{\mathrm{NN}}}=5 \mathrm{TeV}$, J. High Energy Phys. 03 (2016) 133.

[50] J. Adam et al. (ALICE Collaboration), Centrality dependence of $\psi(2 \mathrm{~S})$ suppression in $\mathrm{p}-\mathrm{Pb}$ collisions at $\sqrt{s_{\mathrm{NN}}}=5.02 \mathrm{TeV}$, J. High Energy Phys. 06 (2016) 050.

[51] R. Rapp, Signatures of thermal dilepton radiation at RHIC, Phys. Rev. C 63, 054907 (2001).

[52] R. Rapp, Dilepton spectroscopy of QCD matter at collider energies, Adv. High Energy Phys. 2013, 148253 (2013).

[53] C. Shen, J.-F. Paquet, G. S. Denicol, S. Jeon, and C. Gale, Collectivity and electromagnetic radiation in small systems, Phys. Rev. C 95, 014906 (2017).

[54] B. Abelev et al. (ALICE Collaboration), Performance of the ALICE experiment at the CERN LHC, Int. J. Mod. Phys. A 29, 1430044 (2014).
[55] K. Aamodt et al. (ALICE Collaboration), Alignment of the ALICE inner tracking system with cosmic-ray tracks, JINST 5, P03003 (2010).

[56] J. Alme et al., The ALICE TPC, a large 3-dimensional tracking device with fast readout for ultra-high multiplicity events, Nucl. Instrum. Methods Phys. Res. A 622, 316 (2010).

[57] A. Akindinov et al., Performance of the ALICE Time-OfFlight detector at the LHC, Eur. Phys. J. Plus 128, 44 (2013).

[58] E. Abbas et al. (ALICE Collaboration), Performance of the ALICE VZERO system, JINST 8, P10016 (2013).

[59] ALICE Collaboration, ALICE luminosity determination for $\mathrm{pp}$ collisions at $\sqrt{s}=5 \mathrm{TeV}$, Tech. Rep. No. ALICE-PUBLIC2016-005, CERN LHC, ALICE, 2016, https://cds.cern.ch/ record/2202638.

[60] B. Abelev et al. (ALICE Collaboration), Measurement of visible cross sections in proton-lead collisions at $\sqrt{s_{\mathrm{NN}}}=$ $5.02 \mathrm{TeV}$ in van der Meer scans with the ALICE detector, JINST 9, P11003 (2014).

[61] R. Brun, R. Hagelberg, M. Hansroul, and J. C. Lassalle, Simulation program for particle physics experiments, GEANT: User guide and reference manual, Tech Rep. No. CERN-DD-78-2, CERN, Geneva, 1978 https://cds.cern.ch/record/118715.

[62] R. Brun, F. Bruyant, F. Carminati, S. Giani, M. Maire, A. McPherson, G. Patrick, and L. Urban, GEANT: Detector Description and Simulation Tool (CERN Program Library, CERN, Geneva, 1993), https://cds.cern.ch/record/1082634.

[63] P. Skands, S. Carrazza, and J. Rojo, Tuning PYTHIA 8.1: The Monash 2013 tune, Eur. Phys. J. C 74, 3024 (2014).

[64] T. Sjostrand, S. Mrenna, and P. Z. Skands, A brief introduction to PYTHIA 8.1, Comput. Phys. Commun. 178, 852 (2008).

[65] S. Roesler, R. Engel, and J. Ranft, The Monte Carlo event generator DPMJET-III, in Advanced Monte Carlo for Radiation Physics, Particle Transport Simulation and Applications, edited by A. Kling, F. J. C. Baräo, M. Nakagawa, L. Távora, P. Vaz (Springer, Berlin, Heidelberg, 2000), pp. 1033-1038.

[66] T. Sjostrand, S. Mrenna, and P. Z. Skands, PYTHIA 6.4 physics and manual, J. High Energy Phys. 05 (2006) 026.

[67] T. Pierog, I. Karpenko, J. M. Katzy, E. Yatsenko, and K. Werner, EPOS LHC: Test of collective hadronization with data measured at the CERN Large Hadron Collider, Phys. Rev. C 92, 034906 (2015).

[68] R. Barlow and C. Beeston, Fitting using finite Monte Carlo samples, Comput. Phys. Commun. 77, 219 (1993).

[69] ALICE Collaboration, Momentum transformation matrix for dielectron simulations in $\mathrm{Pb}-\mathrm{Pb}$ collisions at $\sqrt{s_{\mathrm{NN}}}=$ 2.76 TeV, Tech. Rep. No. ALICE-PUBLIC-2017-011, CERN LHC, ALICE, 2017, https://cds.cern.ch/record/2289779.

[70] A. Adare et al. (PHENIX Collaboration), Detailed measurement of the $e^{+} e^{-}$pair continuum in $p+p$ and $\mathrm{Au}+\mathrm{Au}$ collisions at $\sqrt{s_{\mathrm{NN}}}=200 \mathrm{GeV}$ and implications for direct photon production, Phys. Rev. C 81, 034911 (2010).

[71] S. Acharya et al. (ALICE Collaboration), Production of charged pions, kaons, and (anti-)protons in $\mathrm{Pb}-\mathrm{Pb}$ and inelastic pp collisions at $\sqrt{s}_{\mathrm{NN}}=5.02 \mathrm{TeV}$, Phys. Rev. C 101, 044907 (2020).

[72] J. Adam et al. (ALICE Collaboration), Multiplicity dependence of $\pi^{ \pm}, \mathrm{K}$ and (anti-)p production at large transverse momentum in $\mathrm{p}-\mathrm{Pb}$ collisions at $\sqrt{s_{\mathrm{NN}}}=5.02 \mathrm{TeV}$, Phys. Lett. B 760, 720 (2016). 
[73] M. Biyajima, T. Mizoguchi, N. Nakajima, N. Suzuki, and G. Wilk, Modified Hagedorn formula including temperature fluctuation: Estimation of temperatures at RHIC experiments, Eur. Phys. J. C 48, 597 (2006).

[74] S. Acharya et al. (ALICE Collaboration), Evidence of rescattering effect in $\mathrm{Pb}-\mathrm{Pb}$ collisions at the $\mathrm{LHC}$ through production of $\mathrm{K}^{*}(892)^{0}$ and $\phi(1020)$ mesons, Phys. Lett. B 802, 135225 (2020).

[75] J. Adam et al. (ALICE Collaboration), Production of $\mathrm{K}^{*}(892)^{0}$ and $\phi(1020)$ in $\mathrm{p}-\mathrm{Pb}$ collisions at $\sqrt{s_{\mathrm{NN}}}=5.02 \mathrm{TeV}$, Eur. Phys. J. C 76, 245 (2016).

[76] B. Abelev et al. (ALICE Collaboration), $\pi^{0}$ and $\eta$ meson production in proton-proton collisions at $\sqrt{s}=0.9 \mathrm{TeV}$ and $\sqrt{s}=7 \mathrm{TeV}$, Phys. Lett. B 717, 162 (2012).

[77] S. Acharya et al. (ALICE Collaboration), $\pi^{0}$ and $\eta$ meson production in proton-proton collisions at $\sqrt{s}=8 \mathrm{TeV}$, Eur. Phys. J. C 78, 263 (2018).

[78] S. Acharya et al. (ALICE Collaboration), $\pi^{0}$ and $\eta$ meson production in $\mathrm{p}-\mathrm{Pb}$ collisions at $\sqrt{s_{\mathrm{NN}}}=5.02 \mathrm{TeV}$, Eur. Phys. J. C 78, 624 (2018).

[79] R. Baur et al. (CERES Collaboration), First results of the CERES electron pair spectrometer from $\mathrm{p}+\mathrm{Be}, \mathrm{p}+\mathrm{Au}$ and $\mathrm{S}+$ Au collisions, Nucl. Phys. A 566, 87 (1994).

[80] R. Albrecht et al. (WA80 Collaboration), Production of eta mesons in $200-\mathrm{A} / \mathrm{GeV} \mathrm{S}+\mathrm{S}$ and $\mathrm{S}+$ Au reactions, Phys. Lett. B 361, 14 (1995).

[81] P. K. Khandai, P. Shukla, and V. Singh, Meson spectra and $m_{T}$ scaling in $p+p, d+\mathrm{Au}$, and $\mathrm{Au}+\mathrm{p}$ Au collisions at $\sqrt{s_{\mathrm{NN}}}=$ $200 \mathrm{GeV}$, Phys. Rev. C 84, 054904 (2011).

[82] L. Altenkämper, F. Bock, C. Loizides, and N. Schmidt, Applicability of transverse mass scaling in hadronic collisions at energies available at the CERN Large Hadron Collider, Phys. Rev. C 96, 064907 (2017).

[83] S. Acharya et al. (ALICE Collaboration), Inclusive $\mathrm{J} / \psi$ production at mid-rapidity in pp collisions at $\sqrt{s}=5.02 \mathrm{TeV}$, J. High Energy Phys. 10 (2019) 084.

[84] D. Adamova et al. (ALICE Collaboration), $\mathrm{J} / \psi$ production as a function of charged-particle pseudorapidity density in $\mathrm{p}-\mathrm{Pb}$ collisions at $\sqrt{s_{\mathrm{NN}}}=5.02 \mathrm{TeV}$, Phys. Lett. B 776, 91 (2018).

[85] P. Golonka and Z. Was, PHOTOS Monte Carlo: A precision tool for QED corrections in $Z$ and $W$ decays, Eur. Phys. J. C 45, 97 (2006).

[86] P. Z. Skands, Tuning Monte Carlo generators: The Perugia Tunes, Phys. Rev. D 82, 074018 (2010).

[87] P. Nason, A New method for combining NLO QCD with shower Monte Carlo algorithms, J. High Energy Phys. 11 (2004) 040.

[88] S. Frixione, P. Nason, and G. Ridolfi, A Positive-weight nextto-leading-order Monte Carlo for heavy flavour hadroproduction, J. High Energy Phys. 09 (2007) 126.

[89] S. Frixione, P. Nason, and C. Oleari, Matching NLO QCD computations with Parton Shower simulations: the POWHEG method, J. High Energy Phys. 11 (2007) 070.
[90] S. Alioli, P. Nason, C. Oleari, and E. Re, A general framework for implementing NLO calculations in shower Monte Carlo programs: the POWHEG BOX, J. High Energy Phys. 06 (2010) 043.

[91] ALICE Collaboration, Production of $\omega(782)$ in pp collisions at $\sqrt{s}=7 \mathrm{TeV}$, tech. rep. (2018), https://cds. cern.ch/record/ 2316785

[92] S. Acharya et al. (ALICE Collaboration), Production of the $\rho(770)^{0}$ meson in $\mathrm{pp}$ and $\mathrm{Pb}-\mathrm{Pb}$ collisions at $\sqrt{s_{\mathrm{NN}}}=$ 2.76 TeV, Phys. Rev. C 99, 064901 (2019).

[93] L. Gladilin, Fragmentation fractions of $c$ and $b$ quarks into charmed hadrons at LEP, Eur. Phys. J. C 75, 19 (2015).

[94] B. Abelev et al. (ALICE Collaboration), Measurement of electrons from beauty hadron decays in $p p$ collisions at $\sqrt{s}=$ 7 TeV, Phys. Lett. B 721, 13 (2013); 763, 507 (2016).

[95] S. Acharya et al. (ALICE Collaboration), Measurement of D-meson production at mid-rapidity in $\mathrm{pp}$ collisions at $\sqrt{s}=7 \mathrm{TeV}$, Eur. Phys. J. C 77, 550 (2017).

[96] S. Acharya et al. (ALICE Collaboration), $\Lambda_{\mathrm{c}}^{+}$production in pp collisions at $\sqrt{s}=7 \mathrm{TeV}$ and in $\mathrm{p}-\mathrm{Pb}$ collisions at $\sqrt{s_{\mathrm{NN}}}=$ 5.02 TeV, J. High Energy Phys. 04 (2018) 108.

[97] S. Acharya et al. (ALICE Collaboration), Transverse momentum spectra and nuclear modification factors of charged particles in pp, p- $\mathrm{Pb}$, and $\mathrm{Pb}-\mathrm{Pb}$ collisions at the LHC, J. High Energy Phys. 11 (2018) 013.

[98] R. Rapp and E. Shuryak, Thermal dilepton radiation at intermediate masses at the cern-sps, Phys. Lett. B 473, 13 (2000).

[99] ALICE Collaboration, Upgrade of the ALICE time projection chamber, Tech. Rep. CERN-LHCC-2013-020. ALICE-TDR016, CERN LHC, ALICE, 2013, https://cds.cern.ch/record/ 1622286.

[100] ALICE Collaboration, Addendum to the Technical Design Report for the Upgrade of the ALICE Time Projection Chamber, Tech. Rep. CERN-LHCC-2015-002. ALICE-TDR016-ADD-1, CERN LHC, ALICE, 2015, https://cds.cern.ch/ record/1984329.

[101] L. Musa (ALICE Collaboration), Upgrade of the inner tracking system conceptual design report. conceptual design report for the upgrade of the ALICE ITS, Tech. Rep. CERN-LHCC2012-013. LHCC-P-005, CERN, Geneva, 2012, https://cds. cern.ch/record/1475244.

[102] P. Antonioli, A. Kluge, and W. Riegler (ALICE Collaboration), Upgrade of the ALICE readout and trigger system, Tech. Rep. CERN-LHCC-2013-019. ALICE-TDR015, CERN LHC, ALICE, 2013, https://cds.cern.ch/record/ 1603472.

[103] P. Buncic, M. Krzewicki, and P. Vande Vyvre (ALICE Collaboration), Technical design report for the upgrade of the online-offline computing system, Tech. Rep. CERN-LHCC2015-006. ALICE-TDR-019, CERN, Geneva, 2015, https:// cds.cern.ch/record/2011297.

S. Acharya, ${ }^{141}$ D. Adamová, ${ }^{95}$ A. Adler, ${ }^{74}$ J. Adolfsson, ${ }^{81}$ M. M. Aggarwal, ${ }^{100}$ G. Aglieri Rinella, ${ }^{34}$ M. Agnello, ${ }^{30}$ N. Agrawal, ${ }^{10,54}$ Z. Ahammed, ${ }^{141}$ S. Ahmad, ${ }^{16}$ S. U. Ahn, ${ }^{76}$ Z. Akbar, ${ }^{51}$ A. Akindinov, ${ }^{92}$ M. Al-Turany, ${ }^{107}$ S. N. Alam, ${ }^{40,141}$ D. S. D. Albuquerque, ${ }^{122}$ D. Aleksandrov ${ }^{88}$ B. Alessandro, ${ }^{59}$ H. M. Alfanda, ${ }^{6}$ R. Alfaro Molina, ${ }^{71}$ B. Ali, ${ }^{16}$ Y. Ali, ${ }^{14}$ A. Alici, ${ }^{10,26,54}$ N. Alizadehvandchali, ${ }^{125}$ A. Alkin, ${ }^{2,34}$ J. Alme, ${ }^{21}$ T. Alt, ${ }^{68}$ L. Altenkamper, ${ }^{21}$ I. Altsybeev, ${ }^{113}$ M. N. Anaam, 
C. Andrei ${ }^{48}$ D. Andreou, ${ }^{34}$ A. Andronic, ${ }^{144}$ M. Angeletti, ${ }^{34}$ V. Anguelov, ${ }^{104}$ C. Anson, ${ }^{15}$ T. Antičić,,${ }^{108} \mathrm{~F}$. Antinori,,${ }^{57}$ P. Antonioli,${ }^{54}$ N. Apadula ${ }^{80}$ L. Aphecetche, ${ }^{115} \mathrm{H}$. Appelshäuser ${ }^{68}$ S. Arcelli, ${ }^{26}$ R. Arnaldi, ${ }^{59}$ M. Arratia,${ }^{80}$ I. C. Arsene, ${ }^{20}$ M. Arslandok ${ }^{104}$ A. Augustinus, ${ }^{34}$ R. Averbeck,,${ }^{107}$ S. Aziz,${ }^{78}$ M. D. Azmi, ${ }^{16}$ A. Badalà, ${ }^{56}$ Y. W. Baek, ${ }^{41}$ S. Bagnasco,${ }^{59}$ X. Bai, ${ }^{107}$ R. Bailhache, ${ }^{68}$ R. Bala, ${ }^{101}$ A. Balbino, ${ }^{30}$ A. Baldisseri, ${ }^{137}$ M. Ball, ${ }^{43}$ S. Balouza, ${ }^{105}$ D. Banerjee, ${ }^{3}$ R. Barbera, ${ }^{27}$

L. Barioglio, ${ }^{25}$ G. G. Barnaföldi, ${ }^{145}$ L. S. Barnby,${ }^{94}$ V. Barret,${ }^{134}$ P. Bartalini, ${ }^{6}$ C. Bartels, ${ }^{127}$ K. Barth ${ }^{34}$ E. Bartsch, ${ }^{68}$

F. Baruffaldi, ${ }^{28}$ N. Bastid ${ }^{134}$ S. Basu, ${ }^{143}$ G. Batigne, ${ }^{115}$ B. Batyunya,${ }^{75}$ D. Bauri, ${ }^{49}$ J. L. Bazo Alba,,${ }^{12}$ I. G. Bearden,${ }^{89}$

C. Beattie, ${ }^{146}$ C. Bedda,${ }^{63}$ N. K. Behera, ${ }^{61}$ I. Belikov, ${ }^{136}$ A. D. C. Bell Hechavarria, ${ }^{144}$ F. Bellini, ${ }^{34}$ R. Bellwied, ${ }^{125}$

V. Belyaev, ${ }^{93}$ G. Bencedi, ${ }^{145}$ S. Beole,${ }^{25}$ A. Bercuci ${ }^{48}$ Y. Berdnikov, ${ }^{98}$ D. Berenyi, ${ }^{145}$ R. A. Bertens, ${ }^{130}$ D. Berzano, ${ }^{59}$

M. G. Besoiu, ${ }^{67}$ L. Betev, ${ }^{34}$ A. Bhasin, ${ }^{101}$ I. R. Bhat, ${ }^{101}$ M. A. Bhat, ${ }^{3}$ H. Bhatt,,${ }^{49}$ B. Bhattacharjee, ${ }^{42}$ A. Bianchi, ${ }^{25}$ L. Bianchi, ${ }^{25}$

N. Bianchi, ${ }^{52}$ J. Bielčík,,${ }^{37}$ J. Bielč́́ková, ${ }^{95}$ A. Bilandzic, ${ }^{105}$ G. Biro, ${ }^{145}$ R. Biswas, ${ }^{3}$ S. Biswas, ${ }^{3}$ J. T. Blair, ${ }^{119}$ D. Blau, ${ }^{88}$

C. Blume, ${ }^{68}$ G. Boca, ${ }^{139}$ F. Bock,${ }^{96}$ A. Bogdanov, ${ }^{93}$ S. Boi, ${ }^{23}$ J. Bok ${ }^{61}$ L. Boldizsár, ${ }^{145}$ A. Bolozdynya,${ }^{93}$ M. Bombara ${ }^{38}$

G. Bonomi, ${ }^{140}$ H. Borel,${ }^{137}$ A. Borissov, ${ }^{93}$ H. Bossi, ${ }^{146}$ E. Botta ${ }^{25}$ L. Bratrud ${ }^{68}$ P. Braun-Munzinger,${ }^{107}$ M. Bregant, ${ }^{121}$

M. Broz,${ }^{37}$ E. Bruna, ${ }^{59}$ G. E. Bruno, ${ }^{33,106}$ M. D. Buckland, ${ }^{127}$ D. Budnikov, ${ }^{109}$ H. Buesching, ${ }^{68}$ S. Bufalino, ${ }^{30}$ O. Bugnon,,${ }^{115}$

P. Buhler, ${ }^{114}$ P. Buncic, ${ }^{34}$ Z. Buthelezi, ${ }^{72,131}$ J. B. Butt, ${ }^{14}$ S. A. Bysiak,,${ }^{18}$ D. Caffarri, ${ }^{90}$ A. Caliva, ${ }^{107}$ E. Calvo Villar, ${ }^{12}$

J. M. M. Camacho, ${ }^{120}$ R. S. Camacho, ${ }^{45}$ P. Camerini, ${ }^{24}$ F. D. M. Canedo, ${ }^{121}$ A. A. Capon, ${ }^{114}$ F. Carnesecchi, ${ }^{26}$ R. Caron, ${ }^{137}$

J. Castillo Castellanos, ${ }^{137}$ A. J. Castro, ${ }^{130}$ E. A. R. Casula, ${ }^{55}$ F. Catalano, ${ }^{30}$ C. Ceballos Sanchez, ${ }^{75}$ P. Chakraborty, ${ }^{49}$

S. Chandra, ${ }^{141}$ W. Chang, ${ }^{6}$ S. Chapeland, ${ }^{34}$ M. Chartier, ${ }^{127}$ S. Chattopadhyay, ${ }^{141}$ S. Chattopadhyay, ${ }_{110}$ A. Chauvin, ${ }^{23}$

C. Cheshkov, ${ }^{135}$ B. Cheynis, ${ }^{135}$ V. Chibante Barroso, ${ }^{34}$ D. D. Chinellato, ${ }^{122}$ S. Cho, ${ }^{61}$ P. Chochula, ${ }^{34}$ T. Chowdhury, ${ }^{134}$

P. Christakoglou, ${ }^{90}$ C. H. Christensen, ${ }^{89}$ P. Christiansen, ${ }^{81}$ T. Chujo, ${ }^{133}$ C. Cicalo, ${ }^{55}$ L. Cifarelli, ${ }^{10,26}$ L. D. Cilladi, ${ }^{25}$

F. Cindolo, ${ }^{54}$ M. R. Ciupek, ${ }^{107}$ G. Clai, ${ }^{54,}{ }^{*}$ J. Cleymans, ${ }^{124}$ F. Colamaria,,${ }^{53}$ D. Colella, ${ }^{53}$ A. Collu, ${ }^{80}$ M. Colocci, ${ }^{26}$

M. Concas, ${ }^{59,{ }^{\dagger}}$ G. Conesa Balbastre, ${ }^{79}$ Z. Conesa del Valle, ${ }^{78}$ G. Contin, ${ }^{24,60}$ J. G. Contreras, ${ }^{37}$ T. M. Cormier ${ }^{96}$

Y. Corrales Morales, ${ }^{25}$ P. Cortese, ${ }^{31}$ M. R. Cosentino, ${ }^{123}$ F. Costa, ${ }^{34}$ S. Costanza, ${ }^{139}$ P. Crochet, ${ }^{134}$ E. Cuautle, ${ }^{69}$ P. Cui, ${ }^{6}$

L. Cunqueiro ${ }^{96}$ D. Dabrowski, ${ }^{142}$ T. Dahms, ${ }^{105}$ A. Dainese,${ }^{57}$ F. P. A. Damas, $,{ }^{15,}, 137$ M. C. Danisch, ${ }^{104}$ A. Danu, ${ }^{67}$ D. Das, ${ }^{110}$

I. Das, ${ }_{110}$ P. Das, ${ }^{86}$ P. Das, ${ }^{3}$ S. Das, ${ }^{3}$ A. Dash, ${ }^{86}$ S. Dash, ${ }^{49}$ S. De,${ }^{86}$ A. De Caro, ${ }^{29}$ G. de Cataldo, ${ }^{53}$ J. de Cuveland, ${ }^{39}$ A. De

Falco, ${ }^{23}$ D. De Gruttola, ${ }^{10}$ N. De Marco ${ }^{59}$ S. De Pasquale, ${ }^{29}$ S. Deb,${ }^{50}$ H. F. Degenhardt, ${ }^{121}$ K. R. Deja, ${ }^{142}$ A. Deloff, ${ }^{85}$

S. Delsanto, ${ }^{25,131}$ W. Deng, ${ }^{6}$ P. Dhankher, ${ }^{49}$ D. Di Bari, ${ }^{33}$ A. Di Mauro, ${ }^{34}$ R. A. Diaz,${ }^{8}$ T. Dietel, ${ }^{124}$ P. Dillenseger,${ }^{68}$ Y. Ding, ${ }^{6}$

R. Divià, ${ }^{34}$ D. U. Dixit, ${ }^{19} \varnothing$. Djuvsland, ${ }^{21}$ U. Dmitrieva ${ }^{62}$ A. Dobrin, ${ }^{67}$ B. Dönigus, ${ }^{68}$ O. Dordic,${ }^{20}$ A. K. Dubey,,${ }^{141}$

A. Dubla, ${ }^{90,107}$ S. Dudi, ${ }^{100}$ M. Dukhishyam, ${ }^{86}$ P. Dupieux, ${ }^{134}$ R. J. Ehlers, ${ }^{66}$ V. N. Eikeland, ${ }^{21}$ D. Elia,,${ }^{53}$ B. Erazmus, ${ }^{115}$

F. Erhardt, ${ }^{99}$ A. Erokhin, ${ }^{113}$ M. R. Ersdal, ${ }^{21}$ B. Espagnon, ${ }^{78}$ G. Eulisse, ${ }^{34}$ D. Evans, ${ }^{111}$ S. Evdokimov, ${ }^{91}$ L. Fabbietti, ${ }^{105}$

M. Faggin ${ }^{28}$ J. Faivre, ${ }^{79}$ F. Fan, ${ }^{6}$ A. Fantoni, ${ }^{52}$ M. Fasel,${ }^{96}$ P. Fecchio,${ }^{30}$ A. Feliciello, ${ }^{59}$ G. Feofilov, ${ }^{113}$ A. Fernández Téllez,${ }^{45}$

A. Ferrero, ${ }^{137}$ A. Ferretti, ${ }^{25}$ A. Festanti, ${ }^{34}$ V. J. G. Feuillard, ${ }^{104}$ J. Figiel,${ }^{118}$ S. Filchagin, ${ }^{109}$ D. Finogeev, ${ }^{62}$ F. M. Fionda, ${ }^{21}$

G. Fiorenza, ${ }^{53}$ F. Flor, ${ }^{125}$ A. N. Flores, ${ }^{119}$ S. Foertsch, ${ }^{72}$ P. Foka, ${ }^{107}$ S. Fokin, ${ }^{88}$ E. Fragiacomo, ${ }^{60}$ U. Frankenfeld, ${ }^{107}$ U. Fuchs, ${ }^{34}$

C. Furget, ${ }^{79}$ A. Furs, ${ }^{62}$ M. Fusco Girard, ${ }^{29}$ J. J. Gaardh $\varnothing j e,{ }^{89}$ M. Gagliardi, ${ }^{25}$ A. M. Gago, ${ }^{112}$ A. Gal, ${ }^{136}$ C. D. Galvan, ${ }^{120}$

P. Ganoti, ${ }^{84}$ C. Garabatos, ${ }^{107}$ J. R. A. Garcia, ${ }^{45}$ E. Garcia-Solis, ${ }^{11}$ K. Garg, ${ }^{115}$ C. Gargiulo, ${ }^{34}$ A. Garibli, ${ }^{87}$ K. Garner, ${ }^{144}$

P. Gasik, ${ }^{105,107}$ E. F. Gauger, ${ }^{119}$ M. B. Gay Ducati, ${ }^{70}$ M. Germain, ${ }^{115}$ J. Ghosh, ${ }^{110}$ P. Ghosh, ${ }^{141}$ S. K. Ghosh, ${ }^{3}$ M. Giacalone ${ }^{26}$

P. Gianotti, ${ }^{52}$ P. Giubellino, ${ }^{59,107}$ P. Giubilato, ${ }^{28}$ A. M. C. Glaenzer, ${ }^{137}$ P. Glässel, ${ }^{104}$ A. Gomez Ramirez, ${ }^{74}$ V. Gonzalez, ${ }^{107,}{ }^{143}$

L. H. González-Trueba, ${ }^{71}$ S. Gorbunov, ${ }^{39}$ L. Görlich, ${ }^{11}$ A. Goswami, ${ }^{49}$ S. Gotovac, ${ }^{35}$ V. Grabski, ${ }^{71}$ L. K. Graczykowski, ${ }^{142}$

K. L. Graham, ${ }^{111}$ L. Greiner, ${ }^{80}$ A. Grelli, ${ }^{63}$ C. Grigoras, ${ }^{34}$ V. Grigoriev,${ }^{93}$ A. Grigoryan, ${ }^{1}$ S. Grigoryan, ${ }^{75}$ O. S. Groettvik, ${ }^{21}$

F. Grosa ${ }^{30,59}$ J. F. Grosse-Oetringhaus, ${ }^{34}$ R. Grosso, ${ }^{107}$ R. Guernane, ${ }^{79}$ M. Guittiere,,${ }^{115}$ K. Gulbrandsen, ${ }^{89}$ T. Gunji, ${ }^{132}$

A. Gupta, ${ }^{101}$ R. Gupta, ${ }^{101}$ I. B. Guzman, ${ }^{45}$ R. Haake, ${ }^{146}$ M. K. Habib, ${ }^{107}$ C. Hadjidakis,${ }^{78}$ H. Hamagaki, ${ }^{82}$ G. Hamar, ${ }^{145}$

M. Hamid, ${ }^{6}$ R. Hannigan, ${ }^{119}$ M. R. Haque, ${ }^{63,86}$ A. Harlenderova, ${ }^{107}$ J. W. Harris, ${ }^{146}$ A. Harton, ${ }^{11}$ J. A. Hasenbichler, ${ }^{34}$

H. Hassan, ${ }^{96}$ Q. U. Hassan, ${ }^{14}$ D. Hatzifotiadou, ${ }^{10,54}$ P. Hauer, ${ }^{43}$ L. B. Havener, ${ }^{146}$ S. Hayashi, ${ }^{132}$ S. T. Heckel, ${ }^{105}$ E. Hellbär, ${ }^{68}$

H. Helstrup, ${ }^{36}$ A. Herghelegiu, ${ }^{48}$ T. Herman, ${ }^{37}$ E. G. Hernandez, ${ }^{45}$ G. Herrera Corral, ${ }^{9}$ F. Herrmann, ${ }^{144}$ K. F. Hetland, ${ }^{36}$

H. Hillemanns,${ }^{34}$ C. Hills,${ }^{127}$ B. Hippolyte, ${ }^{136}$ B. Hohlweger, ${ }^{105}$ J. Honermann,,${ }^{144}$ D. Horak,${ }^{37}$ A. Hornung, ${ }^{68}$ S. Hornung, ${ }^{107}$

R. Hosokawa, ${ }^{15,133}$ P. Hristov, ${ }^{34}$ C. Huang,${ }^{78}$ C. Hughes,,${ }^{130}$ P. Huhn, ${ }^{68}$ T. J. Humanic, ${ }^{97}$ H. Hushnud,${ }^{110}$ L. A. Husova,,${ }^{144}$

N. Hussain, ${ }^{42}$ S. A. Hussain, ${ }^{14}$ D. Hutter,${ }^{39}$ J. P. Iddon, ${ }^{34,127}$ R. Ilkaev, ${ }^{109}$ H. Ilyas,,${ }^{14}$ M. Inaba, ${ }^{133}$ G. M. Innocenti,,${ }^{34}$

M. Ippolitov, ${ }^{88}$ A. Isakov, ${ }^{95}$ M. S. Islam, ${ }^{110}$ M. Ivanov, ${ }^{107}$ V. Ivanov, ${ }^{98}$ V. Izucheev, ${ }^{11}$ B. Jacak,${ }^{80}$ N. Jacazio,${ }^{34,54}$

P. M. Jacobs, ${ }^{80}$ S. Jadlovska, ${ }^{117}$ J. Jadlovsky, ${ }^{117}$ S. Jaelani, ${ }^{63}$ C. Jahnke, ${ }^{121}$ M. J. Jakubowska, ${ }^{142}$ M. A. Janik, ${ }^{142}$ T. Janson, ${ }^{74}$

M. Jercic, ${ }^{99}$ O. Jevons, ${ }^{111}$ M. Jin, ${ }^{125}$ F. Jonas, ${ }^{96,144}$ P. G. Jones, ${ }^{111}$ J. Jung, ${ }^{68}$ M. Jung, ${ }^{68}$ A. Jusko, ${ }^{111}$ P. Kalinak, ${ }^{64}$ A. Kalweit, ${ }^{34}$

V. Kaplin, ${ }^{93}$ S. Kar, ${ }^{6}$ A. Karasu Uysal, ${ }^{77}$ D. Karatovic, ${ }^{99}$ O. Karavichev, ${ }^{62}$ T. Karavicheva, ${ }^{62}$ P. Karczmarczyk,,${ }^{142}$

E. Karpechev, ${ }^{62}$ A. Kazantsev ${ }^{88}$ U. Kebschull, ${ }^{74}$ R. Keidel, ${ }^{47}$ M. Keil, ${ }^{34}$ B. Ketzer, ${ }^{43}$ Z. Khabanova, ${ }^{90}$ A. M. Khan, ${ }^{6}$ S. Khan, ${ }^{16}$

A. Khanzadeev, ${ }^{98}$ Y. Kharlov, ${ }^{91}$ A. Khatun, ${ }^{16}$ A. Khuntia, ${ }^{118}$ B. Kileng, ${ }^{36}$ B. Kim ${ }^{61}$ B. Kim, ${ }^{133}$ D. Kim, ${ }^{147}$ D. J. Kim, ${ }^{126}$

E. J. Kim, ${ }^{73}$ H. Kim, ${ }^{17}$ J. Kim, ${ }^{147}$ J. S. Kim, ${ }^{41}$ J. Kim, ${ }^{104}$ J. Kim, ${ }^{147}$ J. Kim, ${ }^{73}$ M. Kim, ${ }^{104}$ S. Kim, ${ }^{18}$ T. Kim, ${ }^{147}$ T. Kim, ${ }^{147}$

S. Kirsch ${ }^{68}$ I. Kisel, ${ }^{39}$ S. Kiselev, ${ }^{92}$ A. Kisiel, ${ }^{142}$ J. L. Klay, ${ }^{5}$ C. Klein, ${ }^{68}$ J. Klein, ${ }^{34,59}$ S. Klein, ${ }^{80}$ C. Klein-Bösing, ${ }^{144}$

M. Kleiner, ${ }^{68}$ A. Kluge, ${ }^{34}$ M. L. Knichel, ${ }^{34}$ A. G. Knospe, ${ }^{125}$ C. Kobdaj, ${ }^{116}$ M. K. Köhler, ${ }^{104}$ T. Kollegger, ${ }^{107}$ A. Kondratyev, ${ }^{75}$

N. Kondratyeva, ${ }^{93}$ E. Kondratyuk, ${ }^{91}$ J. Konig, ${ }^{68}$ S. A. Konigstorfer, ${ }^{105}$ P. J. Konopka, ${ }^{34}$ G. Kornakov, ${ }^{142}$ L. Koska, ${ }^{117}$

O. Kovalenko, ${ }^{85}$ V. Kovalenko, ${ }^{113}$ M. Kowalski, ${ }^{118}$ I. Králik,${ }^{64}$ A. Kravčáková, ${ }^{38}$ L. Kreis, ${ }^{107}$ M. Krivda, ${ }^{64,111}$ F. Krizek, ${ }^{95}$

K. Krizkova Gajdosova, ${ }^{37}$ M. Krüger ${ }^{68}$ E. Kryshen, ${ }^{98}$ M. Krzewicki, ${ }^{39}$ A. M. Kubera, ${ }^{97}$ V. Kučera ${ }^{34,61}$ C. Kuhn, ${ }^{136}$ 
P. G. Kuijer, ${ }^{90}$ L. Kumar, ${ }^{100}$ S. Kundu, ${ }^{86}$ P. Kurashvili, ${ }^{85}$ A. Kurepin, ${ }^{62}$ A. B. Kurepin, ${ }^{62}$ A. Kuryakin, ${ }^{109}$ S. Kushpil, ${ }^{95}$ J. Kvapil, ${ }^{111}$ M. J. Kweon, ${ }^{61}$ J. Y. Kwon, ${ }^{61}$ Y. Kwon, ${ }^{147}$ S. L. La Pointe, ${ }^{39}$ P. La Rocca, ${ }^{27}$ Y. S. Lai, ${ }^{80}$ M. Lamanna, ${ }^{34}$ R. Langoy, ${ }^{129}$ K. Lapidus, ${ }^{34}$ A. Lardeux, ${ }^{20}$ P. Larionov, ${ }^{52}$ E. Laudi, ${ }^{34}$ R. Lavicka, ${ }^{37}$ T. Lazareva, ${ }^{113}$ R. Lea, ${ }^{24}$ L. Leardini, ${ }^{104}$ J. Lee, ${ }^{133}$ S. Lee, ${ }^{147}$ S. Lehner, ${ }^{114}$ J. Lehrbach, ${ }^{39}$ R. C. Lemmon, ${ }^{94}$ I. León Monzón, ${ }^{120}$ E. D. Lesser, ${ }^{19}$ M. Lettrich, ${ }^{34}$ P. Lévai, ${ }^{145}$ X. Li,${ }^{12}$ X. L. Li, ${ }^{6}$ J. Lien, ${ }^{129}$ R. Lietava, ${ }^{111}$ B. Lim, ${ }^{17}$ V. Lindenstruth, ${ }^{39}$ A. Lindner, ${ }^{48}$ C. Lippmann, ${ }^{107}$ M. A. Lisa, ${ }^{97}$ A. Liu, ${ }^{19}$ J. Liu, ${ }^{127}$ S. Liu, ${ }^{97}$ W. J. Llope, ${ }^{143}$ I. M. Lofnes,${ }^{21}$ V. Loginov, ${ }^{93}$ C. Loizides,${ }^{96}$ P. Loncar, ${ }^{35}$ J. A. Lopez, ${ }^{104}$ X. Lopez, ${ }^{134}$ E. López Torres, ${ }^{8}$ J. R. Luhder, ${ }^{144}$ M. Lunardon, ${ }^{28}$ G. Luparello, ${ }^{60}$ Y. G. Ma, ${ }^{40}$ A. Maevskaya, ${ }^{62}$ M. Mager, ${ }^{34}$ S. M. Mahmood, ${ }^{20}$ T. Mahmoud, ${ }^{43}$ A. Maire, ${ }^{136}$ R. D. Majka, ${ }^{146, \ddagger}$ M. Malaev, ${ }^{98}$ Q. W. Malik, ${ }^{20}$ L. Malinina, ${ }^{75,8}$ D. Mal' Kevich, ${ }^{92}$ P. Malzacher, ${ }^{107}$ G. Mandaglio, ${ }^{32,56}$ V. Manko, ${ }^{88}$ F. Manso, ${ }^{134}$ V. Manzari, ${ }^{53}$ Y. Mao, ${ }^{6}$ M. Marchisone, ${ }^{135}$ J. Mareš, ${ }^{66}$ G. V. Margagliotti, ${ }^{24}$ A. Margotti, ${ }^{54}$ A. Marín, ${ }^{107}$ C. Markert, ${ }^{119}$ M. Marquard, ${ }^{68}$ C. D. Martin, ${ }^{24}$ N. A. Martin, ${ }^{104}$ P. Martinengo, ${ }^{34}$ J. L. Martinez, ${ }^{125}$ M. I. Martínez, ${ }^{45}$ G. Martínez García, ${ }^{15}$ S. Masciocchi, ${ }^{107}$ M. Masera, ${ }^{25}$ A. Masoni, ${ }^{55}$ L. Massacrier, ${ }^{78}$ E. Masson, ${ }^{115}$ A. Mastroserio, ${ }^{53,138}$ A. M. Mathis, ${ }^{105}$ O. Matonoha, ${ }^{81}$ P. F. T. Matuoka, ${ }^{121}$ A. Matyja, ${ }^{118}$ C. Mayer, ${ }^{118}$ F. Mazzaschi, ${ }^{25}$ M. Mazzilli, ${ }^{53}$ M. A. Mazzoni, ${ }^{58}$ A. F. Mechler, ${ }^{68}$ F. Meddi, ${ }^{22}$ Y. Melikyan, ${ }^{62,93}$

A. Menchaca-Rocha, ${ }^{71}$ C. Mengke, ${ }^{6}$ E. Meninno, ${ }^{29,114}$ A. S. Menon, ${ }^{125}$ M. Meres, ${ }^{13}$ S. Mhlanga, ${ }^{124}$ Y. Miake, ${ }^{133}$ L. Micheletti, ${ }^{25}$ L. C. Migliorin, ${ }^{135}$ D. L. Mihaylov, ${ }^{105}$ K. Mikhaylov, ${ }^{75,92}$ A. N. Mishra, ${ }^{69}$ D. Miśkowiec, ${ }^{107}$ A. Modak, ${ }^{3}$ N. Mohammadi, ${ }^{34}$ A. P. Mohanty, ${ }^{63}$ B. Mohanty, ${ }^{86}$ M. Mohisin Khan, ${ }^{16, \|}$ Z. Moravcova, ${ }^{89}$ C. Mordasini, ${ }^{105}$ D. A. Moreira De Godoy, ${ }^{144}$ L. A. P. Moreno, ${ }^{45}$ I. Morozov, ${ }^{62}$ A. Morsch, ${ }^{34}$ T. Mrnjavac, ${ }^{34}$ V. Muccifora, ${ }^{52}$ E. Mudnic, ${ }^{35}$ D. Mühlheim, ${ }^{144}$ S. Muhuri, ${ }^{141}$ J. D. Mulligan, ${ }^{80}$ A. Mulliri, ${ }^{23,55}$ M. G. Munhoz, ${ }^{121}$ R. H. Munzer, ${ }^{68}$ H. Murakami, ${ }^{132}$ S. Murray, ${ }^{124}$ L. Musa, ${ }^{34}$ J. Musinsky, ${ }^{64}$ C. J. Myers, ${ }^{125}$ J. W. Myrcha, ${ }^{142}$ B. Naik, ${ }^{49}$ R. Nair, ${ }^{85}$ B. K. Nandi, ${ }^{49}$ R. Nania, ${ }^{10,54}$ E. Nappi, ${ }^{53}$ M. U. Naru, ${ }^{14}$ A. F. Nassirpour, ${ }^{81}$ C. Nattrass, ${ }^{130}$ R. Nayak, ${ }^{49}$ T. K. Nayak,${ }^{86}$ S. Nazarenko, ${ }^{109}$ A. Neagu, ${ }^{20}$ R. A. Negrao De Oliveira, ${ }^{68}$

L. Nellen, ${ }^{69}$ S. V. Nesbo, ${ }^{36}$ G. Neskovic, ${ }^{39}$ D. Nesterov, ${ }^{113}$ L. T. Neumann, ${ }^{142}$ B. S. Nielsen, ${ }^{89}$ S. Nikolaev, ${ }^{88}$ S. Nikulin, ${ }^{88}$ V. Nikulin, ${ }^{98}$ F. Noferini, ${ }^{10,54}$ P. Nomokonov, ${ }^{75}$ J. Norman, ${ }^{79,127}$ N. Novitzky, ${ }^{133}$ P. Nowakowski, ${ }^{142}$ A. Nyanin, ${ }^{88}$ J. Nystrand, ${ }^{21}$ M. Ogino, ${ }^{82}$ A. Ohlson, ${ }^{81,104}$ J. Oleniacz, ${ }^{142}$ A. C. Oliveira Da Silva, ${ }^{130}$ M. H. Oliver, ${ }^{146}$ C. Oppedisano, ${ }^{59}$

A. Ortiz Velasquez, ${ }^{69}$ A. Oskarsson, ${ }^{81}$ J. Otwinowski, ${ }^{118}$ K. Oyama, ${ }^{82}$ Y. Pachmayer, ${ }^{104}$ V. Pacik, ${ }^{89}$ S. Padhan, ${ }^{49}$ D. Pagano, ${ }^{140}$ G. Paić, ${ }^{69}$ J. Pan, ${ }^{143}$ S. Panebianco, ${ }^{137}$ P. Pareek, ${ }^{50,141}$ J. Park, ${ }^{61}$ J. E. Parkkila, ${ }^{126}$ S. Parmar, ${ }^{100}$ S. P. Pathak, ${ }^{125}$ B. Paul, ${ }^{23}$ J. Pazzini, ${ }^{140}$ H. Pei, ${ }^{6}$ T. Peitzmann, ${ }^{63}$ X. Peng, ${ }^{6}$ L. G. Pereira, ${ }^{70}$ H. Pereira Da Costa, ${ }^{137}$ D. Peresunko, ${ }^{88}$ G. M. Perez, ${ }^{8}$ S. Perrin, ${ }^{137}$ Y. Pestov, ${ }^{4}$ V. Petráček, ${ }^{37}$ M. Petrovici, ${ }^{48}$ R. P. Pezzi, ${ }^{70}$ S. Piano, ${ }^{60}$ M. Pikna, ${ }^{13}$ P. Pillot, ${ }^{115}$ O. Pinazza, ${ }^{34,54}$ L. Pinsky, ${ }^{125}$ C. Pinto, ${ }^{27}$ S. Pisano, ${ }^{10,52}$ D. Pistone, ${ }^{56}$ M. Płoskoń, ${ }^{80}$ M. Planinic, ${ }^{99}$ F. Pliquett, ${ }^{68}$ M. G. Poghosyan, ${ }^{96}$ B. Polichtchouk, ${ }^{91}$ N. Poljak, ${ }^{99}$ A. Pop, ${ }^{48}$ S. Porteboeuf-Houssais, ${ }^{134}$ V. Pozdniakov, ${ }^{75}$ S. K. Prasad, ${ }^{3}$ R. Preghenella, ${ }^{54}$ F. Prino, ${ }^{59}$ C. A. Pruneau, ${ }^{143}$ I. Pshenichnov, ${ }^{62}$ M. Puccio, ${ }^{34}$ J. Putschke, ${ }^{143}$ S. Qiu, ${ }^{90}$ L. Quaglia, ${ }^{25}$ R. E. Quishpe, ${ }^{125}$ S. Ragoni, ${ }^{111}$ S. Raha, ${ }^{3}$ S. Rajput, ${ }^{101}$ J. Rak, ${ }^{126}$ A. Rakotozafindrabe, ${ }^{137}$ L. Ramello, ${ }^{31}$ F. Rami, ${ }^{136}$ S. A. R. Ramirez, ${ }^{45}$ R. Raniwala, ${ }^{102}$ S. Raniwala, ${ }^{102}$ S. S. Räsänen, ${ }^{44}$ R. Rath, ${ }^{50}$ V. Ratza, ${ }^{43}$ I. Ravasenga, ${ }^{90}$ K. F. Read, ${ }^{96,130}$ A. R. Redelbach, ${ }^{39}$ K. Redlich, ${ }^{85, \pi}$ A. Rehman, ${ }^{21}$ P. Reichelt, ${ }^{68}$ F. Reidt, ${ }^{34}$ X. Ren, ${ }^{6}$ R. Renfordt, ${ }^{68}$ Z. Rescakova, ${ }^{38}$ K. Reygers, ${ }^{104}$ A. Riabov, ${ }^{98}$ V. Riabov, ${ }^{98}$ T. Richert, ${ }^{81,89}$ M. Richter, ${ }^{20}$ P. Riedler, ${ }^{34}$ W. Riegler, ${ }^{34}$ F. Riggi, ${ }^{27}$ C. Ristea, ${ }^{67}$ S. P. Rode, ${ }^{50}$

M. Rodríguez Cahuantzi, ${ }^{45}$ K. Røed, ${ }^{20}$ R. Rogalev, ${ }^{91}$ E. Rogochaya, ${ }^{75}$ D. Rohr, ${ }^{34}$ D. Röhrich, ${ }^{21}$ P. F. Rojas, ${ }^{45}$ P. S. Rokita, ${ }^{142}$ F. Ronchetti, ${ }^{52}$ A. Rosano, ${ }^{56}$ E. D. Rosas, ${ }^{69}$ K. Roslon, ${ }^{142}$ A. Rossi, ${ }^{28,57}$ A. Rotondi, ${ }^{139}$ A. Roy, ${ }^{50}$ P. Roy, ${ }^{110}$ O. V. Rueda, ${ }^{81}$ R. Rui, ${ }^{24}$ B. Rumyantsev, ${ }^{75}$ A. Rustamov, ${ }^{87}$ E. Ryabinkin, ${ }^{88}$ Y. Ryabov, ${ }^{98}$ A. Rybicki, ${ }^{118}$ H. Rytkonen, ${ }^{126}$

O. A. M. Saarimaki, ${ }^{44}$ R. Sadek, ${ }^{115}$ S. Sadhu, ${ }^{141}$ S. Sadovsky, ${ }^{91}$ K. Šafař́k, ${ }^{37}$ S. K. Saha, ${ }^{141}$ B. Sahoo, ${ }^{49}$ P. Sahoo, ${ }^{49}$ R. Sahoo, ${ }^{50}$ S. Sahoo, ${ }^{65}$ P. K. Sahu, ${ }^{65}$ J. Saini, ${ }^{141}$ S. Sakai, ${ }^{133}$ S. Sambyal, ${ }^{101}$ V. Samsonov, ${ }^{93,98}$ D. Sarkar, ${ }^{143}$ N. Sarkar, ${ }^{141}$ P. Sarma, ${ }^{42}$ V. M. Sarti, ${ }^{105}$ M. H. P. Sas, ${ }^{63}$ E. Scapparone,${ }^{54}$ J. Schambach, ${ }^{119}$ H. S. Scheid, ${ }^{68}$ C. Schiaua, ${ }^{48}$ R. Schicker, ${ }^{104}$ A. Schmah, ${ }^{104}$

C. Schmidt, ${ }^{107}$ H. R. Schmidt, ${ }^{103}$ M. O. Schmidt, ${ }^{104}$ M. Schmidt, ${ }^{103}$ N. V. Schmidt, ${ }^{68,96}$ A. R. Schmier, ${ }^{130}$ J. Schukraft,${ }^{89}$ Y. Schutz, ${ }^{136}$ K. Schwarz, ${ }^{107}$ K. Schweda, ${ }^{107}$ G. Scioli, ${ }^{26}$ E. Scomparin, ${ }^{59}$ J. E. Seger, ${ }^{15}$ Y. Sekiguchi, ${ }^{132}$ D. Sekihata, ${ }^{132}$ I. Selyuzhenkov, ${ }^{93,107}$ S. Senyukov, ${ }^{136}$ D. Serebryakov, ${ }^{62}$ A. Sevcenco, ${ }^{67}$ A. Shabanov, ${ }^{62}$ A. Shabetai, ${ }^{115}$ R. Shahoyan, ${ }^{34}$ W. Shaikh, ${ }^{110}$ A. Shangaraev, ${ }^{91}$ A. Sharma, ${ }^{100}$ A. Sharma, ${ }^{101}$ H. Sharma, ${ }^{118}$ M. Sharma, ${ }^{101}$ N. Sharma, ${ }^{100}$ S. Sharma, ${ }^{101}$ O. Sheibani, ${ }^{125}$ K. Shigaki, ${ }^{46}$ M. Shimomura, ${ }^{83}$ S. Shirinkin, ${ }^{92}$ Q. Shou, ${ }^{40}$ Y. Sibiriak, ${ }^{88}$ S. Siddhanta, ${ }^{55}$ T. Siemiarczuk, ${ }^{85}$ D. Silvermyr, ${ }^{11}$ G. Simatovic, ${ }^{90}$ G. Simonetti, ${ }^{34}$ B. Singh, ${ }^{105}$ R. Singh, ${ }^{86}$ R. Singh, ${ }^{101}$ R. Singh, ${ }^{50}$ V. K. Singh, ${ }^{141}$ V. Singhal, ${ }^{141}$ T. Sinha, ${ }^{110}$ B. Sitar, ${ }^{13}$ M. Sitta, ${ }^{31}$ T. B. Skaali, ${ }^{20}$ M. Slupecki, ${ }^{44}$ N. Smirnov, ${ }^{146}$ R. J. M. Snellings, ${ }^{63}$ C. Soncco, ${ }^{112}$ J. Song, ${ }^{125}$ A. Songmoolnak, ${ }^{116}$ F. Soramel, ${ }^{28}$ S. Sorensen, ${ }^{130}$ I. Sputowska, ${ }^{118}$ J. Stachel, ${ }^{104}$ I. Stan, ${ }^{67}$ P. J. Steffanic, ${ }^{130}$ E. Stenlund, ${ }^{81}$

S. F. Stiefelmaier, ${ }^{104}$ D. Stocco, ${ }^{115}$ M. M. Storetvedt, ${ }^{36}$ L. D. Stritto, ${ }^{29}$ A. A. P. Suaide, ${ }^{121}$ T. Sugitate, ${ }^{46}$ C. Suire, ${ }^{78}$

M. Suleymanov, ${ }^{14}$ M. Suljic, ${ }^{34}$ R. Sultanov, ${ }^{92}$ M. Šumbera, ${ }^{95}$ V. Sumberia, ${ }^{101}$ S. Sumowidagdo, ${ }^{51}$ S. Swain, ${ }^{65}$ A. Szabo, ${ }^{13}$ I. Szarka, ${ }^{13}$ U. Tabassam, ${ }^{14}$ S. F. Taghavi, ${ }^{105}$ G. Taillepied, ${ }^{134}$ J. Takahashi, ${ }^{122}$ G. J. Tambave,${ }^{21}$ S. Tang, ${ }^{6,134}$ M. Tarhini, ${ }^{115}$ M. G. Tarzila, ${ }^{48}$ A. Tauro, ${ }^{34}$ G. Tejeda Muñoz ${ }^{45}$ A. Telesca, ${ }^{34}$ L. Terlizzi, ${ }^{25}$ C. Terrevoli, ${ }^{125}$ D. Thakur,${ }^{50}$ S. Thakur, ${ }^{141}$ D. Thomas, ${ }^{119}$ F. Thoresen, ${ }^{89}$ R. Tieulent, ${ }^{135}$ A. Tikhonov, ${ }^{62}$ A. R. Timmins, ${ }^{125}$ A. Toia, ${ }^{68}$ N. Topilskaya, ${ }^{62}$ M. Toppi, ${ }^{52}$ F. Torales-Acosta, ${ }^{19}$ S. R. Torres, ${ }^{37}$ A. Trifiró, ${ }^{32,56}$ S. Tripathy, ${ }^{50,69}$ T. Tripathy, ${ }^{49}$ S. Trogolo, ${ }^{28}$ G. Trombetta, ${ }^{33}$ L. Tropp, ${ }^{38}$ V. Trubnikov, ${ }^{2}$ W. H. Trzaska, ${ }^{126}$ T. P. Trzcinski, ${ }^{142}$ B. A. Trzeciak, ${ }^{37,63}$ A. Tumkin, ${ }^{109}$ R. Turrisi, ${ }^{57}$ T. S. Tveter, ${ }^{20}$

K. Ullaland, ${ }^{21}$ E. N. Umaka, ${ }^{125}$ A. Uras, ${ }^{135}$ G. L. Usai, ${ }^{23}$ M. Vala, ${ }^{38}$ N. Valle, ${ }^{139}$ S. Vallero, ${ }^{59}$ N. van der Kolk, ${ }^{63}$ L. V. R. van

Doremalen, ${ }^{63}$ M. van Leeuwen, ${ }^{63}$ P. Vande Vyvre, ${ }^{34}$ D. Varga, ${ }^{145}$ Z. Varga, ${ }^{145}$ M. Varga-Kofarago, ${ }^{145}$ A. Vargas, ${ }^{45}$

M. Vasileiou, ${ }^{84}$ A. Vasiliev, ${ }^{88}$ O. Vázquez Doce, ${ }^{105}$ V. Vechernin, ${ }^{113}$ E. Vercellin, ${ }^{25}$ S. Vergara Limón, ${ }^{45}$ L. Vermunt, ${ }^{63}$ 
R. Vernet, ${ }^{7}$ R. Vértesi, ${ }^{145}$ L. Vickovic, ${ }^{35}$ L. H. Viebach, ${ }^{68}$ Z. Vilakazi, ${ }^{131}$ O. Villalobos Baillie, ${ }^{111}$ G. Vino, ${ }^{53}$ A. Vinogradov ${ }^{88}$ T. Virgili, ${ }^{29}$ V. Vislavicius, ${ }^{89}$ A. Vodopyanov ${ }^{75}$ B. Volkel,,${ }^{34}$ M. A. Völkl,${ }^{103}$ K. Voloshin,,${ }^{92}$ S. A. Voloshin, ${ }^{143}$ G. Volpe,${ }^{33}$ B. von Haller, ${ }^{34}$ I. Vorobyev ${ }^{105}$ D. Voscek,,${ }^{117}$ J. Vrláková, ${ }^{38}$ B. Wagner,${ }^{21}$ M. Weber, ${ }^{114}$ S. G. Weber, ${ }^{144}$ A. Wegrzynek, ${ }^{34}$ S. C. Wenzel, ${ }^{34}$ J. P. Wessels, ${ }^{144}$ J. Wiechula, ${ }^{68}$ J. Wikne, ${ }^{20}$ G. Wilk,${ }^{85}$ J. Wilkinson, ${ }^{10,54}$ G. A. Willems, ${ }^{144}$ E. Willsher, ${ }^{111}$ B. Windelband, ${ }^{104}$ M. Winn, ${ }^{137}$ W. E. Witt, ${ }^{130}$ J. R. Wright, ${ }^{119}$ Y. Wu, ${ }^{128}$ R. Xu, ${ }^{6}$ S. Yalcin, ${ }^{77}$ Y. Yamaguchi, ${ }^{46}$ K. Yamakawa, ${ }^{46}$ S. Yang ${ }^{21}$ S. Yano ${ }^{137}$ Z. Yin, ${ }^{6}$ H. Yokoyama ${ }^{63}$ I.-K. Yoo, ${ }^{17}$ J. H. Yoon, ${ }^{61}$ S. Yuan, ${ }^{21}$ A. Yuncu, ${ }^{104}$ V. Yurchenko, ${ }^{2}$ V. Zaccolo, ${ }^{24}$ A. Zaman, ${ }^{14}$ C. Zampolli, ${ }^{34}$ H. J. C. Zanoli, ${ }^{63}$ N. Zardoshti, ${ }^{34}$ A. Zarochentsev,,${ }^{13}$ P. Závada ${ }^{66}$ N. Zaviyalov,,${ }^{109}$ H. Zbroszczyk, ${ }^{142}$ M. Zhalov, ${ }^{98}$ S. Zhang, ${ }^{40}$ X. Zhang, ${ }^{6}$ Z. Zhang, ${ }^{6}$ V. Zherebchevskii, ${ }^{113}$ Y. Zhi, ${ }^{12}$ D. Zhou, ${ }^{6}$ Y. Zhou, ${ }^{89}$ Z. Zhou, ${ }^{21}$ J. Zhu, ${ }^{6,107}$ Y. Zhu, ${ }^{6}$ A. Zichichi, ${ }^{10,26}$ G. Zinovjev, ${ }^{2}$ and N. Zurlo ${ }^{140}$

\title{
(ALICE Collaboration)
}

\author{
${ }^{1}$ A. I. Alikhanyan National Science Laboratory (Yerevan Physics Institute) Foundation, Yerevan, Armenia \\ ${ }^{2}$ Bogolyubov Institute for Theoretical Physics, National Academy of Sciences of Ukraine, Kiev, Ukraine \\ ${ }^{3}$ Bose Institute, Department of Physics and Centre for Astroparticle Physics and Space Science (CAPSS), Kolkata, India \\ ${ }^{4}$ Budker Institute for Nuclear Physics, Novosibirsk, Russia \\ ${ }^{5}$ California Polytechnic State University, San Luis Obispo, California, USA \\ ${ }^{6}$ Central China Normal University, Wuhan, China \\ ${ }^{7}$ Centre de Calcul de l'IN2P3, Villeurbanne, Lyon, France \\ ${ }^{8}$ Centro de Aplicaciones Tecnológicas y Desarrollo Nuclear (CEADEN), Havana, Cuba \\ ${ }^{9}$ Centro de Investigación y de Estudios Avanzados (CINVESTAV), Mexico City and Mérida, Mexico \\ ${ }^{10}$ Centro Fermi - Museo Storico della Fisica e Centro Studi e Ricerche "Enrico Fermi', Rome, Italy \\ ${ }^{11}$ Chicago State University, Chicago, Illinois, USA \\ ${ }^{12}$ China Institute of Atomic Energy, Beijing, China \\ ${ }^{13}$ Comenius University Bratislava, Faculty of Mathematics, Physics and Informatics, Bratislava, Slovakia \\ ${ }^{14}$ COMSATS University Islamabad, Islamabad, Pakistan \\ ${ }^{15}$ Creighton University, Omaha, Nebraska, USA \\ ${ }^{16}$ Department of Physics, Aligarh Muslim University, Aligarh, India \\ ${ }^{17}$ Department of Physics, Pusan National University, Pusan, Republic of Korea \\ ${ }^{18}$ Department of Physics, Sejong University, Seoul, Republic of Korea \\ ${ }^{19}$ Department of Physics, University of California, Berkeley, California, USA \\ ${ }^{20}$ Department of Physics, University of Oslo, Oslo, Norway \\ ${ }^{21}$ Department of Physics and Technology, University of Bergen, Bergen, Norway \\ ${ }^{22}$ Dipartimento di Fisica dell'Università 'La Sapienza' and Sezione INFN, Rome, Italy \\ ${ }^{23}$ Dipartimento di Fisica dell'Università and Sezione INFN, Cagliari, Italy \\ ${ }^{24}$ Dipartimento di Fisica dell'Università and Sezione INFN, Trieste, Italy \\ ${ }^{25}$ Dipartimento di Fisica dell'Università and Sezione INFN, Turin, Italy \\ ${ }^{26}$ Dipartimento di Fisica e Astronomia dell'Università and Sezione INFN, Bologna, Italy \\ ${ }^{27}$ Dipartimento di Fisica e Astronomia dell'Università and Sezione INFN, Catania, Italy \\ ${ }^{28}$ Dipartimento di Fisica e Astronomia dell'Università and Sezione INFN, Padova, Italy \\ ${ }^{29}$ Dipartimento di Fisica 'E. R. Caianiello' dell'Università and Gruppo Collegato INFN, Salerno, Italy \\ ${ }^{30}$ Dipartimento DISAT del Politecnico and Sezione INFN, Turin, Italy \\ ${ }^{31}$ Dipartimento di Scienze e Innovazione Tecnologica dell'Università del Piemonte Orientale and INFN Sezione di Torino, Alessandria, Italy \\ ${ }^{32}$ Dipartimento di Scienze MIFT, Università di Messina, Messina, Italy \\ ${ }^{33}$ Dipartimento Interateneo di Fisica 'M. Merlin' and Sezione INFN, Bari, Italy \\ ${ }^{34}$ European Organization for Nuclear Research (CERN), Geneva, Switzerland \\ ${ }^{35}$ Faculty of Electrical Engineering, Mechanical Engineering and Naval Architecture, University of Split, Split, Croatia \\ ${ }^{36}$ Faculty of Engineering and Science, Western Norway University of Applied Sciences, Bergen, Norway \\ ${ }^{37}$ Faculty of Nuclear Sciences and Physical Engineering, Czech Technical University in Prague, Prague, Czech Republic \\ ${ }^{38}$ Faculty of Science, P. J. Šafárik University, Košice, Slovakia \\ ${ }^{39}$ Frankfurt Institute for Advanced Studies, Johann Wolfgang Goethe-Universität Frankfurt, Frankfurt, Germany \\ ${ }^{40}$ Fudan University, Shanghai, China \\ ${ }^{41}$ Gangneung-Wonju National University, Gangneung, Republic of Korea \\ ${ }^{42}$ Gauhati University, Department of Physics, Guwahati, India \\ ${ }^{43}$ Helmholtz-Institut für Strahlen- und Kernphysik, Rheinische Friedrich-Wilhelms-Universität Bonn, Bonn, Germany \\ ${ }^{44}$ Helsinki Institute of Physics (HIP), Helsinki, Finland \\ ${ }^{45}$ High Energy Physics Group, Universidad Autónoma de Puebla, Puebla, Mexico \\ ${ }^{46}$ Hiroshima University, Hiroshima, Japan
}


${ }^{47}$ Hochschule Worms, Zentrum für Technologietransfer und Telekommunikation (ZTT), Worms, Germany

${ }^{48}$ Horia Hulubei National Institute of Physics and Nuclear Engineering, Bucharest, Romania

${ }^{49}$ Indian Institute of Technology Bombay (IIT), Mumbai, India

${ }^{50}$ Indian Institute of Technology Indore, Indore, India

${ }^{51}$ Indonesian Institute of Sciences, Jakarta, Indonesia

${ }^{52}$ INFN, Laboratori Nazionali di Frascati, Frascati, Italy

${ }^{53}$ INFN, Sezione di Bari, Bari, Italy

${ }^{54}$ INFN, Sezione di Bologna, Bologna, Italy

${ }^{55}$ INFN, Sezione di Cagliari, Cagliari, Italy

${ }^{56}$ INFN, Sezione di Catania, Catania, Italy

${ }^{57}$ INFN, Sezione di Padova, Padova, Italy

${ }^{58}$ INFN, Sezione di Roma, Rome, Italy

${ }^{59}$ INFN, Sezione di Torino, Turin, Italy

${ }^{60}$ INFN, Sezione di Trieste, Trieste, Italy

${ }^{61}$ Inha University, Incheon, Republic of Korea

${ }^{62}$ Institute for Nuclear Research, Academy of Sciences, Moscow, Russia

${ }^{63}$ Institute for Subatomic Physics, Utrecht University/Nikhef, Utrecht, Netherlands

${ }^{64}$ Institute of Experimental Physics, Slovak Academy of Sciences, Košice, Slovakia

${ }^{65}$ Institute of Physics, Homi Bhabha National Institute, Bhubaneswar, India

${ }^{66}$ Institute of Physics of the Czech Academy of Sciences, Prague, Czech Republic

${ }^{67}$ Institute of Space Science (ISS), Bucharest, Romania

${ }^{68}$ Institut für Kernphysik, Johann Wolfgang Goethe-Universität Frankfurt, Frankfurt, Germany

${ }^{69}$ Instituto de Ciencias Nucleares, Universidad Nacional Autónoma de México, Mexico City, Mexico

${ }^{70}$ Instituto de Física, Universidade Federal do Rio Grande do Sul (UFRGS), Porto Alegre, Brazil

${ }^{71}$ Instituto de Física, Universidad Nacional Autónoma de México, Mexico City, Mexico

${ }^{72}$ iThemba LABS, National Research Foundation, Somerset West, South Africa

${ }^{73}$ Jeonbuk National University, Jeonju, Republic of Korea

${ }^{74}$ Johann-Wolfgang-Goethe Universität Frankfurt Institut für Informatik, Fachbereich Informatik und Mathematik, Frankfurt, Germany

${ }^{75}$ Joint Institute for Nuclear Research (JINR), Dubna, Russia

${ }^{76}$ Korea Institute of Science and Technology Information, Daejeon, Republic of Korea

${ }^{77}$ KTO Karatay University, Konya, Turkey

${ }^{78}$ Laboratoire de Physique des 2 Infinis, Irène Joliot-Curie, Orsay, France

${ }^{79}$ Laboratoire de Physique Subatomique et de Cosmologie, Université Grenoble-Alpes, CNRS-IN2P3, Grenoble, France

${ }^{80}$ Lawrence Berkeley National Laboratory, Berkeley, California, USA

${ }^{81}$ Lund University Department of Physics, Division of Particle Physics, Lund, Sweden

${ }^{82}$ Nagasaki Institute of Applied Science, Nagasaki, Japan

${ }^{83}$ Nara Women's University (NWU), Nara, Japan

${ }^{84}$ National and Kapodistrian University of Athens, School of Science, Department of Physics, Athens, Greece

${ }^{85}$ National Centre for Nuclear Research, Warsaw, Poland

${ }^{86}$ National Institute of Science Education and Research, Homi Bhabha National Institute, Jatni, India

${ }^{87}$ National Nuclear Research Center, Baku, Azerbaijan

${ }^{88}$ National Research Centre Kurchatov Institute, Moscow, Russia

${ }^{89}$ Niels Bohr Institute, University of Copenhagen, Copenhagen, Denmark

${ }^{90}$ Nikhef, National institute for subatomic physics, Amsterdam, Netherlands

${ }^{91}$ NRC Kurchatov Institute IHEP, Protvino, Russia

${ }^{92}$ NRC «Kurchatov»Institute - ITEP, Moscow, Russia

${ }^{93}$ NRNU Moscow Engineering Physics Institute, Moscow, Russia

${ }^{94}$ Nuclear Physics Group, STFC Daresbury Laboratory, Daresbury, United Kingdom

${ }^{95}$ Nuclear Physics Institute of the Czech Academy of Sciences, Řěz u Prahy, Czech Republic

${ }^{96}$ Oak Ridge National Laboratory, Oak Ridge, Tennessee, USA

${ }^{97}$ Ohio State University, Columbus, Ohio, USA

${ }^{98}$ Petersburg Nuclear Physics Institute, Gatchina, Russia

${ }^{99}$ Physics department, Faculty of science, University of Zagreb, Zagreb, Croatia

${ }^{100}$ Physics Department, Panjab University, Chandigarh, India

${ }^{101}$ Physics Department, University of Jammu, Jammu, India

${ }^{102}$ Physics Department, University of Rajasthan, Jaipur, India

${ }^{103}$ Physikalisches Institut, Eberhard-Karls-Universität Tübingen, Tübingen, Germany

${ }^{104}$ Physikalisches Institut, Ruprecht-Karls-Universität Heidelberg, Heidelberg, Germany

${ }^{105}$ Physik Department, Technische Universität München, Munich, Germany 
${ }^{106}$ Politecnico di Bari, Bari, Italy

${ }^{107}$ Research Division and ExtreMe Matter Institute EMMI, GSI Helmholtzzentrum für Schwerionenforschung GmbH, Darmstadt, Germany

${ }^{108}$ Rudjer Bošković Institute, Zagreb, Croatia

${ }^{109}$ Russian Federal Nuclear Center (VNIIEF), Sarov, Russia

${ }^{110}$ Saha Institute of Nuclear Physics, Homi Bhabha National Institute, Kolkata, India

${ }^{111}$ School of Physics and Astronomy, University of Birmingham, Birmingham, United Kingdom

${ }^{112}$ Sección Física, Departamento de Ciencias, Pontificia Universidad Católica del Perú, Lima, Peru

${ }^{113}$ St. Petersburg State University, St. Petersburg, Russia

${ }^{114}$ Stefan Meyer Institut für Subatomare Physik (SMI), Vienna, Austria

${ }^{115}$ SUBATECH, IMT Atlantique, Université de Nantes, CNRS-IN2P3, Nantes, France

${ }^{116}$ Suranaree University of Technology, Nakhon Ratchasima, Thailand

${ }^{117}$ Technical University of Košice, Košice, Slovakia

${ }^{118}$ The Henryk Niewodniczanski Institute of Nuclear Physics, Polish Academy of Sciences, Cracow, Poland

${ }^{119}$ The University of Texas at Austin, Austin, Texas, USA

${ }^{120}$ Universidad Autónoma de Sinaloa, Culiacán, Mexico

${ }^{121}$ Universidade de São Paulo (USP), São Paulo, Brazil

${ }^{122}$ Universidade Estadual de Campinas (UNICAMP), Campinas, Brazil

${ }^{123}$ Universidade Federal do ABC, Santo Andre, Brazil

${ }^{124}$ University of Cape Town, Cape Town, South Africa

${ }^{125}$ University of Houston, Houston, Texas, USA

${ }^{126}$ University of Jyväskylä, Jyväskylä, Finland

${ }^{127}$ University of Liverpool, Liverpool, United Kingdom

${ }^{128}$ University of Science and Technology of China, Hefei, China

${ }^{129}$ University of South-Eastern Norway, Tonsberg, Norway

${ }^{130}$ University of Tennessee, Knoxville, Tennessee, USA

${ }^{131}$ University of the Witwatersrand, Johannesburg, South Africa

${ }^{132}$ University of Tokyo, Tokyo, Japan

${ }^{133}$ University of Tsukuba, Tsukuba, Japan

${ }^{134}$ Université Clermont Auvergne, CNRS/IN2P3, LPC, Clermont-Ferrand, France

${ }^{135}$ Université de Lyon, Université Lyon 1, CNRS/IN2P3, IPN-Lyon, Villeurbanne, Lyon, France

${ }^{136}$ Université de Strasbourg, CNRS, IPHC UMR 7178, F-67000 Strasbourg, France, Strasbourg, France

${ }^{137}$ Université Paris-Saclay Centre d'Etudes de Saclay (CEA), IRFU, Départment de Physique Nucléaire (DPhN), Saclay, France

${ }^{138}$ Università degli Studi di Foggia, Foggia, Italy

${ }^{139}$ Università degli Studi di Pavia, Pavia, Italy

${ }^{140}$ Università di Brescia, Brescia, Italy

${ }^{141}$ Variable Energy Cyclotron Centre, Homi Bhabha National Institute, Kolkata, India

${ }^{142}$ Warsaw University of Technology, Warsaw, Poland

${ }^{143}$ Wayne State University, Detroit, Michigan, USA

${ }^{144}$ Westfälische Wilhelms-Universität Münster, Institut für Kernphysik, Münster, Germany

${ }^{145}$ Wigner Research Centre for Physics, Budapest, Hungary

${ }^{146}$ Yale University, New Haven, Connecticut, USA

${ }^{147}$ Yonsei University, Seoul, Republic of Korea

*Italian National Agency for New Technologies, Energy and Sustainable Economic Development (ENEA), Bologna, Italy.

Dipartimento DET del Politecnico di Torino, Turin, Italy.

Deceased.

${ }^{\S}$ M. V. Lomonosov Moscow State University, D. V. Skobeltsyn Institute of Nuclear, Physics, Moscow, Russia.

"Department of Applied Physics, Aligarh Muslim University, Aligarh, India.

IInstitute of Theoretical Physics, University of Wroclaw, Poland. 